\title{
Moving basepoints and the induced automorphisms of link Floer homology
}

\author{
SUCHARIT SARKAR
}

\begin{abstract}
Given an $l$-component pointed oriented link $(L, p)$ in an oriented three-manifold $Y$, one can construct its link Floer chain complex $C F L(Y, L, p)$ over the polynomial ring $\mathbb{F}_{2}\left[U_{1}, \ldots, U_{l}\right]$. Moving the basepoint $p_{i} \in L_{i}$ once around the link component $L_{i}$ induces an automorphism of $\operatorname{CFL}(Y, L, p)$. We study a (possibly different) automorphism of $C F L(Y, L, p)$ defined explicitly in terms of holomorphic disks; for links in $S^{3}$, we show that these two automorphisms are the same.
\end{abstract}

57M25; 57M27, 57R58

\section{Introduction}

Heegaard Floer theory is a collection of invariants, originally defined for pointed oriented closed three-manifolds by Ozsváth and Szabó [12;13] and subsequently extended for pointed oriented knots (by Ozsváth and Szabó [11] and Rasmussen [17]) and pointed oriented links by Ozsváth and Szabó [15] in oriented three-manifolds. For each of these objects, the theory comes in several variants; in each variant, one constructs a chain complex in the graded homotopy category over some graded ring; furthermore, for each object and in each variant, the mapping class group of the object acts on the chain complex.

In this paper, we will work with links. Initially, we will study links in arbitrary oriented three-manifolds; later on, we will concentrate on links in $S^{3}$. Let us first specify the version of link Floer theory that we will study: it is the associated graded object of the minus version of the fully filtered theory over the base ring $\mathbb{F}_{2}\left[U_{1}, \ldots, U_{l}\right]$. We choose to work with the associated graded object and not the (more general) fully filtered theory because the former object is easier to study and also because the proof of Theorem 1.1 is simpler. For simplicity again, we only work with the minus version $C F L^{-}$and not all three of the standard versions $C F L^{-}, C F L^{+}, C F L^{\infty}$ (the general object that we could have studied being the inclusion $\left.\iota: C F L^{-} \hookrightarrow C F L^{\infty}\right)$; however, we can construct $C F L^{\infty}$ and $C F L^{+}$formally from $C F L^{-}$as $C F L^{-} \otimes_{\mathbb{F}_{2}\left[U_{1}, \ldots, U_{l}\right]} \mathbb{F}_{2}\left[U_{1}, U_{1}^{-1}, \ldots, U_{l}, U_{l}^{-1}\right]$ and the mapping cone of $C F L^{-} \rightarrow C F L^{-} \otimes_{\mathbb{F}_{2}\left[U_{1}, \ldots, U_{l}\right]} \mathbb{F}_{2}\left[U_{1}, U_{1}^{-1}, \ldots, U_{l}, U_{l}^{-1}\right]$, 
respectively. We work over the ring $\mathbb{F}_{2}\left[U_{1}, \ldots, U_{l}\right]$ and not the more universal ring $\mathbb{Z}\left[U_{1}, \ldots, U_{l}\right]$ because most of the variants of link Floer theory are only defined over the base field $\mathbb{F}_{2}$ (and, again, because working over $\mathbb{F}_{2}$ is simpler). Therefore, henceforth whenever we say $C F$ or $C F L$, we mean the associated graded object of the minus version of the link Floer chain complex over $\mathbb{F}_{2}\left[U_{1}, \ldots, U_{l}\right]$. (We will give precise definitions of some of these objects in the next section.)

In Section 2, we will give a quick tour of the relevant areas of Heegaard Floer theory. We will describe how to represent an $l$-component pointed link $(L, p)$ in a three-manifold $Y$ by a Heegaard diagram $\mathcal{H}$, and then how to construct a chain complex $C F_{\left(\mathcal{H}, J_{s}\right)}$, viewed as an object in a certain homotopy category $K\left(\mathcal{A}_{l}\right)$, in terms of the Heegaard diagram $\mathcal{H}$ and some additional data $J_{S}$; the link-invariant $C F L(Y, L, p)$, viewed as an object in an equivalent category $N\left(K\left(\mathcal{A}_{l}\right)\right)$, can be obtained from $C F_{\left(\mathcal{H}, J_{s}\right)}$ using naturality; see Ozsváth and Szabó [14], Ozsváth and Stipsicz [9], Juhász [2] and Juhász and Thurton [3]. In Section 3, we will study the mapping class group action $\rho: \operatorname{MCG}(Y, L, p) \rightarrow \operatorname{Aut}_{N\left(K\left(\mathcal{A}_{l}\right)\right)}(C F L(Y, L, p))$ and a specific mapping class group element $\sigma_{i} \in \operatorname{MCG}(Y, L, p)$, the positive Dehn twist around the $i^{\text {th }}$ link component $L_{i}$, which corresponds to moving the basepoint $p_{i} \in L_{i}$ once around. In Section 4, we will define certain link-invariant maps $\Phi_{i}$ and $\Psi_{i}$ from $C F_{\left(\mathcal{H}, J_{s}\right)}$ to itself using counts of certain holomorphic disks and prove that the map $\mathrm{Id}+\Phi_{i} \Psi_{i}$ induces a well-defined automorphism of $C F L(Y, L, p)$. Our main theorem is the following:

Theorem 1.1 Let $\mathcal{H}=(\Sigma, \alpha, \beta, z, w)$ be a Heegaard diagram representing an $l-$ component pointed link $(L, p)$ in $S^{3}$; then, for all $1 \leq i \leq l$, the automorphism Id $+\Psi_{i} \Phi_{i}$ in $\operatorname{Aut}_{K\left(\mathcal{A}_{l}\right)}\left(C F_{\left(\mathcal{H}, J_{s}\right)}\right)$ induces $\rho\left(\sigma_{i}\right)$ in $\operatorname{Aut}_{N\left(K\left(\mathcal{A}_{l}\right)\right)}\left(C F L\left(S^{3}, L, p\right)\right)$, where $\sigma_{i} \in \operatorname{MCG}\left(S^{3}, L, p\right)$ is the positive Dehn twist along the $i^{\text {th }}$ link component $L_{i}$ and $\rho\left(\sigma_{i}\right)$ is its induced automorphism on $\operatorname{CFL}\left(S^{3}, L, p\right)$.

In Section 5, we will use grid diagrams to prove Theorem 1.1; and finally in Section 6, we will compute the automorphism Id $+\Phi_{1} \Psi_{1}$ for all the 85 prime knots up to nine crossings and see that it is non-trivial (as in, not the identity) more often than not.

\section{Acknowledgments}

The author was supported by the Clay Mathematics Institute Postdoctoral Fellowship when this paper was written. He would like to thank Elisenda Grigsby, András Juhász, Robert Lipshitz, Peter Ozsváth, Morwen Thistlethwaite and Zoltán Szabó for many helpful discussions and the referee for several comments and corrections. 


\section{Heegaard Floer basics}

A pointed link is a link with a basepoint in each component. Let $L \subset Y$ be an oriented $l$-component pointed link inside a closed, oriented three-manifold $Y$; let $L_{i}$ be the $i^{\text {th }}$ component and let $p_{i} \in L_{i}$ be the basepoint in the $i^{\text {th }}$ component. We say that $\mathcal{H}=(\Sigma, \alpha, \beta, z, w)$ is a Heegaard diagram for $L$ if there exists a self-indexing Morse function $f: Y \rightarrow \mathbb{R}$, equipped with a gradient-like flow, such that:

- $\Sigma=f^{-1}\left(\frac{3}{2}\right)$ is a surface of genus $g$.

- $z=\left(z_{1}, \ldots, z_{l}\right), w=\left(w_{1}, \ldots, w_{l}\right)$ and there is an $l$-tuple $k=\left(k_{1}, \ldots, k_{l}\right)$ such that $z_{i}$ is a collection of $k_{i}$ markings $z_{i, 1}, \ldots, z_{i, k_{i}}$ in $\Sigma$ and $w_{i}$ is also a collection of $k_{i}$ markings $w_{i, 1}, \ldots, w_{i, k_{i}}$ in $\Sigma$.

- For each $i \in\{1, \ldots, l\}$, one of the $w_{i}$-markings, say $w_{i, s_{i}}$, is designated special.

- $f$ has $|k|$ index-zero critical points and $|k|$ index-three critical points.

- $\alpha$ is the intersection of $\Sigma$ and the stable manifold of the index-one critical points.

- $\beta$ is the intersection of $\Sigma$ and the unstable manifold of the index-two critical points.

- $L_{i}$ is the union of the flowlines through the $z_{i}$-markings and the reversed flowlines through the $w_{i}$-markings.

- The basepoint $p_{i} \in L_{i}$ is the special $w_{i}$-marking $w_{i, s_{i}}$.

- The Heegaard diagram is also assumed to be admissible [15, Definition 3.5].

Let $n=g+|k|-1$ and let $\mathfrak{j}$ be a complex structure on $\Sigma ; J_{s}$ is a path of nearly symmetric, almost complex structures on the symmetric product $\operatorname{Sym}^{n}(\Sigma)$, which is a generic perturbation of the constant path $\operatorname{Sym}^{n}(j)$ [13, Definition 3.1]; $\mathbb{T}_{\alpha}=$ $\left\{x \in \operatorname{Sym}^{n}(\Sigma) \mid\right.$ all the coordinates of $x$ lie on $\left.\alpha\right\}$ is a totally real, half-dimensional torus; $\mathbb{T}_{\beta}$ is defined similarly; the marking $z_{i, j}$ gives rise to the divisor $Z_{i, j}=$ $\left\{x \in \operatorname{Sym}^{n}(\Sigma) \mid\right.$ at least one of the coordinates of $x$ is $\left.z_{i, j}\right\} ; W_{i, j}$ is defined similarly; let $Z_{i}=\sum_{j} Z_{i, j}, W_{i}=\sum_{j} W_{i, j}, Z=\sum_{i} Z_{i}$ and $W=\sum_{i} W_{i}$.

Given $x, y \in \mathbb{T}_{\alpha} \cap \mathbb{T}_{\beta}, \pi_{2}(x, y)$ is the set of all Whitney disks joining $x$ to $y$ or, in other words, the set of all homotopy classes of maps

$\left(\{z \in \mathbb{C}|| z \mid \leq 1\},\left\{i e^{i \theta} \mid-\frac{1}{2} \pi \leq \theta \leq \frac{1}{2} \pi\right\},\left\{e^{i \theta} \mid-\frac{1}{2} \pi \leq \theta \leq \frac{1}{2} \pi\right\}, i,-i\right)$

$\longrightarrow\left(\operatorname{Sym}^{n}(\Sigma), \mathbb{T}_{\alpha}, \mathbb{T}_{\beta}, x, y\right) ;$

given a Whitney disk $\varphi \in \pi_{2}(x, y), \hat{\mathcal{M}}_{J_{s}}(\varphi)$ is the unparametrized moduli space of such maps. Elements of $\mathbb{T}_{\alpha} \cap \mathbb{T}_{\beta}$ carry a Maslov grading $M$ [14, Theorem 7.1] 
and $l$ Alexander gradings $A_{i}\left[15\right.$, Section 8.1] such that, whenever $\varphi \in \pi_{2}(x, y)$, $M(y)-M(x)=\mu(\varphi)-2 \varphi \cdot W$ and $A_{i}(x)-A_{i}(y)=\varphi \cdot\left(Z_{i}-W_{i}\right)$, where the Maslov index $\mu(\varphi)$ is the expected dimension the moduli space $\mathcal{M}_{J_{s}}(\varphi)$. (Here, and throughout the rest of the paper, for any divisor $D$ viewed as a codimension-2 submanifold and for any Whitney disk $\varphi$ viewed as 2 -dimensional submanifold, $\varphi \cdot D$ denotes their algebraic intersection number.) Let $\mathbb{P}$ be the $(l+1)$-graded polynomial ring generated over $\mathbb{F}_{2}$ by the variables $U_{i, j}$ for $i \in\{1, \ldots, l\}, j \in\left\{1, \ldots, k_{i}\right\}$, where the $\left(M, A_{1}, \ldots, A_{l}\right)$ grading of $U_{i, j}$ is $\left(-2,-\delta_{1 i}, \ldots,-\delta_{l i}\right) .{ }^{1}$ The chain complex $C F_{\left(\mathcal{H}, J_{s}\right)}$ is the $(l+1)$-graded $\mathbb{F}_{2}\left[U_{1}, \ldots, U_{l}\right]$-module freely generated over $\mathbb{P}$ by $\mathbb{T}_{\alpha} \cap \mathbb{T}_{\beta}$, where the $U_{i}$-action is multiplication by $U_{i, s_{i}}$; the boundary map is a $U_{i, j}$-equivariant $(-1,0, \ldots, 0)$-graded map and, for $x \in \mathbb{T}_{\alpha} \cap \mathbb{T}_{\beta}$, it is given by

$$
\partial x=\sum_{y \in \mathbb{T}_{\alpha} \cap \mathbb{T}_{\beta}} y \sum_{\begin{array}{c}
\varphi \in \pi_{2}(x, y) \\
\varphi \cdot Z=0 \\
\mu(\varphi)=1
\end{array}}\left|\hat{\mathcal{M}}_{J_{s}}(\varphi)\right| \prod_{l, j} U_{l, j}^{\varphi \cdot W_{l, J}} .
$$

Given a small abelian category $\mathcal{C}$, let $K(\mathcal{C})$ be the homotopy category of chain complexes whose objects are chain complexes in $\mathcal{C}$ and whose morphisms are chain maps up to chain homotopy. Given a small category $\mathcal{C}$ and a group $G$, let $\mathcal{C}_{G}$ be the category whose objects are two-tuples $(A, f)$, where $A \in \mathrm{Ob}_{\mathcal{C}}$ and $f: G \rightarrow \operatorname{Aut}_{\mathcal{C}}(A)$, and the set of morphisms $\operatorname{Mor}_{\mathcal{C}_{G}}\left(\left(A_{1}, f_{1}\right),\left(A_{2}, f_{2}\right)\right)$ is the subset of $\operatorname{Mor}_{\mathcal{C}}\left(A_{1}, A_{2}\right)$ consisting of the ones that are $G$-equivariant. Given a small category $\mathcal{C}$, let $N(\mathcal{C})$ be the category whose objects are three-tuples $\left(I, \mathfrak{o}_{I}, \mathfrak{f}_{I}\right)$, where $I$ is a set, $\mathfrak{o}_{I}$ is a map from $I$ to $\operatorname{Ob}_{\mathcal{C}}$ and $\mathfrak{f}_{I}$ is a map from $I \times I$ to $\operatorname{Mor}_{\mathcal{C}}$, such that $\mathfrak{f}_{I}\left(i, i^{\prime}\right) \in \operatorname{Mor}_{\mathcal{C}}\left(\mathfrak{o}_{I}(i), \mathfrak{o}_{I}\left(i^{\prime}\right)\right)$ for all $i, i^{\prime} \in I, \mathfrak{f}_{I}(i, i)=\operatorname{Id}_{\mathfrak{o}_{I}(i)}$ for all $i \in I$, and $\mathfrak{f}_{I}\left(i^{\prime}, i^{\prime \prime}\right) \mathfrak{f}_{I}\left(i, i^{\prime}\right)=\mathfrak{f}_{I}\left(i, i^{\prime \prime}\right)$ for all $i, i^{\prime}, i^{\prime \prime} \in I$. A morphism from $\left(I, \mathfrak{o}_{I}, \mathfrak{f}_{I}\right)$ to $\left(J, \mathfrak{o}_{J}, \mathfrak{f}_{J}\right)$ is a map $\phi$ from $I \times J$ to $\operatorname{Mor}_{\mathcal{C}}$ such that $\phi(i, j) \in \operatorname{Mor}_{\mathcal{C}}\left(\mathfrak{o}_{I}(i), \mathfrak{o}_{J}(j)\right)$ for all $i \in I$ and all $j \in J$, $\phi\left(i^{\prime}, j\right) \mathfrak{f}_{I}\left(i, i^{\prime}\right)=\phi(i, j)$ for all $i, i^{\prime} \in I$ and all $j \in J$, and $\mathfrak{f}_{J}\left(j, j^{\prime}\right) \phi(i, j)=\phi\left(i, j^{\prime}\right)$ for all $i \in I$ and all $j, j^{\prime} \in J$.

If $\mathcal{A}_{l}$ is the category of $\left(A_{1}, \ldots, A_{l}\right)$-graded $\mathbb{F}_{2}\left[U_{1}, \ldots, U_{l}\right]$-modules, then $C F_{\left(\mathcal{H}, J_{s}\right)}$ is an object of $K\left(\mathcal{A}_{l}\right)$. If $\mathcal{H}^{\prime}=\left(\Sigma^{\prime}, \alpha^{\prime}, \beta^{\prime}, z^{\prime}, w^{\prime}\right)$ is another Heegaard diagram for the same pointed link $(L, p)$ and $J_{s}^{\prime}$ is a path of nearly symmetric, almost complex structures on $\operatorname{Sym}^{n^{\prime}}\left(\Sigma^{\prime}\right)$, then by naturality - see [14, Theorem $2.1 ; 9$, Section $6 ; 2$, Section 5.2; 3, Theorem 1.8] - there is a $U_{i}$-equivariant chain map from $C F_{\left(\mathcal{H}, J_{S}\right)}$ to $C F_{\left(\mathcal{H}^{\prime}, J_{s}^{\prime}\right)}$. It can be checked that this map is well defined up to $U_{i}$-equivariant chain homotopy or, in other words, this map induces a well-defined morphism $F_{\left(\mathcal{H}, J_{s}\right),\left(\mathcal{H}^{\prime}, J_{s}^{\prime}\right)}$ in $K\left(\mathcal{A}_{l}\right)$. Therefore, given a pointed link $(L, p)$ in $Y$, we get a well-defined object $C F L(Y, L, p)$ in $N\left(K\left(\mathcal{A}_{l}\right)\right)$, where the indexing set $I$ is the set of all ordered

\footnotetext{
${ }^{1}$ Throughout the paper, $\delta$ denotes the Kronecker delta function.
} 
pairs $\left(\mathcal{H}, J_{S}\right)$, where $\mathcal{H}$ is a Heegaard diagram for $L$ and $J_{S}$ is a path of nearly symmetric, almost complex structures on the symmetric product, $\mathfrak{o}_{I}\left(\left(\mathcal{H}, J_{S}\right)\right)$ is the chain complex $C F_{\left(\mathcal{H}, J_{s}\right)}$, and $\mathfrak{f}_{I}\left(\left(\mathcal{H}, J_{s}\right),\left(\mathcal{H}^{\prime}, J_{s}^{\prime}\right)\right)$ is the morphism $F_{\left(\mathcal{H}, J_{s}\right),\left(\mathcal{H}^{\prime}, J_{s}^{\prime}\right)}$.

Let $\mathcal{B}_{l}$ be the category of $\left(M, A_{1}, \ldots, A_{l}\right)$-graded $\mathbb{F}_{2}\left[U_{1}, \ldots, U_{l}\right]$-modules and let $\mathcal{C}_{l}$ be the category of $\left(M, A_{1}, \ldots, A_{l}\right)$-graded $\mathbb{F}_{2}$-modules. By taking homology, we get a pointed link-invariant object $\operatorname{HFL}(Y, L, p)=H_{*}(C F L(Y, L, p))$ in $N\left(\mathcal{B}_{l}\right)$; after putting $U_{i}=0$ for all $1 \leq i \leq l$ and then taking homology, we get a pointed link-invariant object $\widehat{H F} L(Y, L, p)=H_{*}\left(C F L(Y, L, p) /\left\{U_{i}=0\right\}\right)$ in $N\left(\mathcal{C}_{l}\right)$. We sometimes need the shift functors in $K\left(\mathcal{A}_{l}\right), \mathcal{B}_{l}$ and $\mathcal{C}_{l}$ (and the induced shift functors in $N\left(K\left(\mathcal{A}_{l}\right)\right), N\left(\mathcal{B}_{l}\right)$ and $\left.N\left(\mathcal{C}_{l}\right)\right)$. Let $\left[m, a_{1}, \ldots, a_{l}\right]$ be the shift functor in any of these categories that decreases the $\left(M, A_{1}, \ldots, A_{l}\right)$-grading by $\left(m, a_{1}, \ldots, a_{l}\right)$.

Remark 2.1 Most of the naturality statements in $[14 ; 9 ; 2 ; 3]$ are stated after taking homology, that is, in the categories $\mathcal{B}_{l}$ or $\mathcal{C}_{l}$. However, the proofs go through almost verbatim in the more general homotopy category $K\left(\mathcal{A}_{l}\right)$ as well.

\section{Mapping class group actions}

Diffeomorphisms will always be orientation preserving, ${ }^{2}$ and the mapping class group MCG is the $\pi_{0}$ of the space of all (orientation-preserving) self-diffeomorphisms. There exists a well-defined map $\rho: \operatorname{MCG}(Y, L, p) \rightarrow \operatorname{Aut}_{N\left(K\left(\mathcal{A}_{l}\right)\right)}(C F L(Y, L, p))$, defined in [14; 9, Definition 6.5; 2, Corollary 5.20; 3, Corollary 1.7] as follows. Let $\sigma \in \operatorname{MCG}(Y, L, p)$; assume that $\sigma$ comes from $\widetilde{\sigma} \in \operatorname{Diff}(Y, L, p)$; let $\widetilde{\sigma}$ also denote the induced automorphisms of the set of all Heegaard diagrams for $(Y, L, p)$, their symmetric products, and the space of all paths of nearly symmetric, almost complex structures on the symmetric products. Therefore, we have a $U_{i, j}$-equivariant chain map from $C F_{\left(\widetilde{\sigma}^{-1}(\mathcal{H}), \tilde{\sigma}^{-1}\left(J_{s}\right)\right)}$ to $C F_{\left(\mathcal{H}, J_{s}\right)}$ which sends $x \in \tilde{\sigma}^{-1}\left(\mathbb{T}_{\alpha}\right) \cap \tilde{\sigma}^{-1}\left(\mathbb{T}_{\beta}\right)$ to $\tilde{\sigma}(x) \in \mathbb{T}_{\alpha} \cap \mathbb{T}_{\beta}$. This induces a well-defined morphism $f_{\sigma}$ from $C F_{\left(\tilde{\sigma}^{-1}(\mathcal{H}), \tilde{\sigma}^{-1}\left(J_{s}\right)\right)}$ to $C F_{\left(\mathcal{H}, J_{S}\right)}$ in $K\left(\mathcal{A}_{l}\right)$ and hence a well-defined automorphism $f_{\sigma} F_{\left(\mathcal{H}, J_{S}\right),\left(\widetilde{\sigma}^{-1}(\mathcal{H}), \widetilde{\sigma}^{-1}\left(J_{s}\right)\right)}$ in $\operatorname{Aut}_{K\left(\mathcal{A}_{l}\right)}\left(C F_{\left(\mathcal{H}, J_{s}\right)}\right)$. It turns out that the maps of the form $F_{\left(\mathcal{H}, J_{s}\right),\left(\mathcal{H}^{\prime}, J_{s}^{\prime}\right)}$ commute with such automorphisms. Therefore, we can treat $C F_{\left(\mathcal{H}, J_{S}\right)}$ as an object in $K\left(\mathcal{A}_{l}\right)_{\mathrm{MCG}(Y, L, p)}$ and the maps $F_{\left(\mathcal{H}, J_{s}\right),\left(\mathcal{H}, J_{s}\right)}$ as morphisms in this category. Therefore, $C F L(Y, L, p)$ can be thought of as a pointed link-invariant object in the category $N\left(K\left(\mathcal{A}_{l}\right)\right)_{\mathrm{MCG}(Y, L, p)}$.

\footnotetext{
${ }^{2}$ In particular, unless otherwise mentioned, a diffeomorphism of the pair $(Y, L)$ is a diffeomorphism that preserves the orientations of both $Y$ and $L$.
} 
Let $T(L)=\prod_{i} L_{i}$ be the pointed $l$-dimensional torus with the basepoint $p=$ $\left(p_{1}, \ldots, p_{l}\right)$. Since $L$ is oriented, $\pi_{1}(T(L), p)$ is canonically isomorphic to $\mathbb{Z}^{l}$. We have a fiber bundle

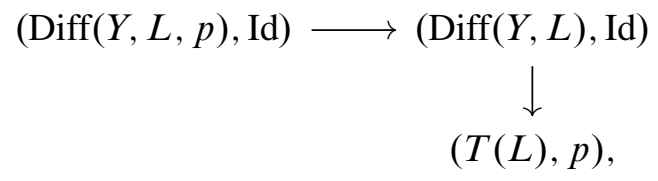

which gives rise to a long exact sequence

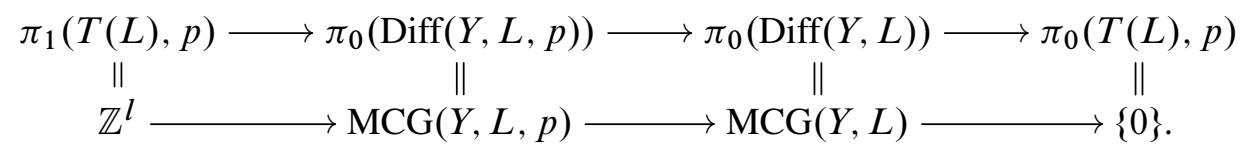

Let $\sigma_{i} \in \operatorname{MCG}(Y, L, p)$ be the image of the $i^{\text {th }}$ unit vector in $\mathbb{Z}^{l}$; we call $\sigma_{i}$ the positive Dehn twist around $L_{i}$. Then there is an action of $\operatorname{MCG}(Y, L)$ on $C F L(Y, L, p)$, which is well defined up to the $l$ automorphisms $\rho\left(\sigma_{i}\right)$. In this paper, we will try to understand these $l$ automorphisms.

Let us first describe a way to view $\rho\left(\sigma_{i}\right)$ as a composition of two triangle maps. Let $\mathcal{H}_{\alpha \beta}=(\Sigma, \alpha, \beta, z, w)$ be a Heegaard diagram for $L$ such that the $i^{\text {th }}$ link component $L_{i}$ contains exactly one $w$ marking and exactly one $z$ marking. By stabilizing twice if necessary, we can assume that there is an oriented arc joining $z_{i, 1}$ to $w_{i, 1}$ that is disjoint from $\alpha$ and intersects $\beta$ transversely at a point, that there is an oriented arc joining $w_{i, 1}$ to $z_{i, 1}$ that is disjoint from $\beta$ and intersects $\alpha$ transversely at a point, and that the union of these two arcs is an oriented embedded circle $C$ on the Heegaard surface $\Sigma$. A regular neighborhood $\operatorname{nbd}(C)$ of $C$ is shown in Figure 3.1.

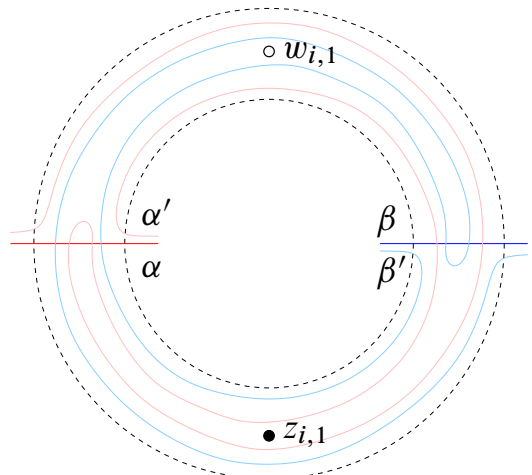

Figure 3.1: The annular neighborhood $\operatorname{nbd}(C)$ 
Let $\partial(\operatorname{nbd}(C))=C_{1}-C_{2}$ with each $C_{i}$ oriented parallel to $C$. Let $\sigma \in \operatorname{Diff}(\Sigma, z, w)$ be the composition of a positive Dehn twist along $C_{1}$ and a negative Dehn twist along $C_{2}$. Let $J_{s}$ be a path of nearly symmetric, almost complex structures on $\operatorname{Sym}^{n}(\Sigma)$ and let $J_{s, t}$ be a path in the space of paths of nearly symmetric, almost complex structures joining $\sigma^{-1}\left(J_{s}\right)$ to $J_{s}$. Let $\alpha^{\prime}$ be obtained by first perturbing $\alpha$ and then applying $\sigma^{-1} ; \beta^{\prime}$ is defined similarly. The multicurves $\alpha, \beta, \alpha^{\prime}$ and $\beta^{\prime}$ in $\operatorname{nbd}(C)$ are represented in Figure 3.1 by the red, blue, pink and light blue curves, respectively. Let $\mathcal{H}_{\alpha \beta^{\prime}}=\left(\Sigma, \alpha, \beta^{\prime}, z, w\right)$ and $\mathcal{H}_{\alpha^{\prime} \beta^{\prime}}=\left(\Sigma, \alpha^{\prime}, \beta^{\prime}, z, w\right)$.

The $\operatorname{MCG}(Y, L, p)$ element induced by $\sigma$ is $\sigma_{i}$. Therefore, the automorphism $\rho\left(\sigma_{i}\right)$ acts by mapping $x \in \mathbb{T}_{\alpha^{\prime}} \cap \mathbb{T}_{\beta^{\prime}}$ in $C F_{\left(\mathcal{H}_{\alpha^{\prime} \beta^{\prime}}, \sigma^{-1}\left(J_{s}\right)\right)}$ to $\sigma(x) \in \mathbb{T}_{\alpha} \cap \mathbb{T}_{\beta}$ in $C F_{\left(\mathcal{H}_{\alpha \beta}, J_{s}\right)}$. The naturality map $F_{\left(\mathcal{H}_{\alpha \beta}, J_{S}\right),\left(\mathcal{H}_{\alpha^{\prime} \beta^{\prime}}, \sigma^{-1}\left(J_{s}\right)\right)}$, by naturality, is the composition

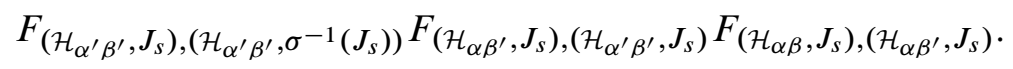

The first two maps are the triangle maps [13, Equation (21)] and it is easily verified that the relevant triple Heegaard diagrams are admissible. The third map is induced by the path $J_{s, t}$ [13, Equation (14)]; however, if we assume that $\hat{M}_{J_{s, t}}(\varphi)$ is empty for all $t \in[0,1]$ and for all Whitney disks $\varphi$ with $\mu(\varphi) \leq 0$, for example by assuming that $\mathcal{H}_{\alpha \beta}$ is a nice Heegaard diagram [19, Definition 3.1], then the third map is the identity map. Therefore, the automorphism $\rho\left(\sigma_{i}\right)$ can be thought of as a composition of two triangle maps.

\section{A candidate}

Following the notations from Section 2 , let $\mathcal{H}=(\Sigma, \alpha, \beta, z, w)$ be a Heegaard diagram for a pointed link $(L, p)$ in $Y$ and let $J_{s}$ be a path of nearly symmetric, almost complex structures on $\operatorname{Sym}^{n}(\Sigma)$. For each $1 \leq i \leq l$, let $a_{i}, b_{i}:\left\{1, \ldots, k_{i}\right\} \rightarrow\left\{1, \ldots, k_{i}\right\}$ be the two bijections such that, for every $j, z_{i, j}$ and $w_{i, a_{i}(j)}$ lie in the same component of $\Sigma \backslash \alpha$ and $z_{i, j}$ and $w_{i, b_{i}(j)}$ lie in the same component of $\Sigma \backslash \beta$. For each $(i, j)$, let us define two $\mathbb{P}$-module maps $\Psi_{i, j}$ and $\Phi_{i, j}$ from $C F_{\left(\mathcal{H}, J_{s}\right)}$ to $C F_{\left(\mathcal{H}, J_{s}\right)}\left[-1,-\delta_{1 i}, \ldots,-\delta_{l i}\right]$ and $C F_{\left(\mathcal{H}, J_{s}\right)}\left[1, \delta_{1 i}, \ldots, \delta_{l i}\right]$, respectively, as follows: For $x \in \mathbb{T}_{\alpha} \cap \mathbb{T}_{\beta}$,

$$
\begin{gathered}
\Psi_{i, j}(x)=\sum_{y \in \mathbb{T}_{\alpha} \cap \mathbb{T}_{\beta}} y \sum_{\substack{\varphi \in \pi_{2}(x, y) \\
\varphi \cdot Z=\varphi \cdot Z_{i, j}=1 \\
\mu(\varphi)=1}}\left|\hat{\mathcal{M}}_{J_{s}}(\varphi)\right| \prod_{l, j} U_{l, j}^{\varphi \cdot W_{l, j}}, \\
\Phi_{i, j}(x)=\sum_{y \in \mathbb{T}_{\alpha} \cap \mathbb{T}_{\beta}} y \sum_{\substack{\varphi \in \pi_{2}(x, y) \\
\varphi \cdot Z=0 \\
\mu(\varphi)=1}}\left(\varphi \cdot W_{i, j}\right)\left|\hat{\mathcal{M}}_{J_{s}}(\varphi)\right| U_{i, j}^{-1} \prod_{l, j} U_{l, j}^{\varphi \cdot W_{l, j}} .
\end{gathered}
$$


A few points are in order. Here, and throughout the paper, we face a scarcity of indexing notations and we usually use $l$ and $j$ as indices. These are different from $i$ and $j$, although, informally speaking, $l$ will "correspond" to $i$ and $j$ will "correspond" to $j$. Secondly, the $U_{i, j}^{-1}$ appearing here (and in a couple of places later on) might look unnatural at first sight since our base ring $\mathbb{P}$ is a polynomial ring over the $U_{l, j}$-variables and does not contain $U_{i, j}^{-1}$. However, this is not an issue since we multiply the term by $\left(\varphi \cdot W_{i, j}\right)$, thereby discarding all Whitney disks $\varphi$ with zero intersection with $W_{i, j}$. In other words, in order to contribute, $\varphi$ has to intersect $W_{i, j}$ at least once, thus picking up a $U_{i, j}$ power, which cancels with $U_{i, j}^{-1}$.

Lemma 4.1 For every $x \in \mathbb{T}_{\alpha} \cap \mathbb{T}_{\beta}$ and every $(i, j)$, the commutators satisfy $\left[\partial: \Psi_{i, j}\right]=U_{i, a_{i}(j)}+U_{i, b_{i}(j)}$ and $\left[\partial: \Phi_{i, j}\right]=0$.

Proof Let us first set up a few extra notations. For any $x \in \mathbb{T}_{\alpha}$, let $\pi_{2}^{\alpha}(x)$ be the set of all Whitney disks with boundary lying in $\mathbb{T}_{\alpha}$ or, in other words, the set of all homotopy classes of maps $(\{z \in \mathbb{C}|| z \mid \leq 1\},\{z \in \mathbb{C}|| z \mid=1\}, i) \rightarrow\left(\operatorname{Sym}^{n}(\Sigma), \mathbb{T}_{\alpha}, x\right)$; given $\varphi^{\alpha} \in \pi_{2}^{\alpha}(x), \hat{\mathcal{N}}_{J_{S}}^{\alpha}(\varphi)$ is the unparametrized moduli space of such maps. The Whitney disks $\pi_{2}^{\beta}(x)$ and the moduli spaces $\hat{\mathcal{N}}_{J_{s}}^{\beta}$ are defined similarly. For every $x \in \mathbb{T}_{\alpha}$ and every $(i, j)$ with $1 \leq i \leq l, 1 \leq j \leq k_{i}$, there exists a unique Whitney disk $\varphi_{x, i, j}^{\alpha} \in \pi_{2}^{\alpha}(x)$ such that $\mu\left(\varphi_{x, i, j}^{\alpha}\right)=2$ and $\varphi_{x, i, j}^{\alpha} \cdot Z_{l, j}$ is 1 if $(l, j)=(i, j)$ and is 0 otherwise; also, $\varphi_{x, i, j}^{\alpha} \cdot W_{l, j}$ is 1 if $(l, j)=\left(i, a_{i}(j)\right)$ and is 0 otherwise; furthermore, if $\varphi^{\alpha} \in \pi_{2}^{\alpha}(x)$ with $\mu\left(\varphi^{\alpha}\right)=2$, then $\hat{\mathcal{N}}_{J_{s}}^{\alpha}\left(\varphi^{\alpha}\right)$ has an odd number of points if and only if $|k|>1$ and $\varphi^{\alpha}=\varphi_{x, i, j}^{\alpha}$ for some $(i, j)$ [15, Theorem 5.5]. Similarly, for every $x \in \mathbb{T}_{\beta}$ and every $(i, j)$, there exists a unique Whitney disk $\varphi_{x, i, j}^{\beta} \in \pi_{2}^{\beta}(x)$ such that $\mu\left(\varphi_{x, i, j}^{\beta}\right)=2$ and $\varphi_{x, i, j}^{\beta} \cdot Z_{l, j}$ is 1 if $(l, j)=(i, j)$ and is 0 otherwise; also, $\varphi_{x, i, j}^{\beta} \cdot W_{l, j}$ is 1 if $(l, j)=\left(i, b_{i}(j)\right)$ and is 0 otherwise; furthermore, if $\varphi^{\beta} \in \pi_{2}^{\beta}(x)$ with $\mu\left(\varphi^{\beta}\right)=2$, then $\hat{\mathcal{N}}_{J_{s}}^{\beta}\left(\varphi^{\beta}\right)$ has an odd number of points if and only if $|k|>1$ and $\varphi^{\beta}=\varphi_{x, i, j}^{\beta}$ for some $(i, j)$.

From Gromov compactification adapted to our present settings [15, Section 6], we know that for any $\varphi \in \pi_{2}(x, y)$ with $\mu(\varphi)=2$, the number of broken flowlines, $\sum_{\varphi=\varphi_{1} * \varphi_{2}}\left|\hat{\mathcal{M}}_{J_{s}}\left(\varphi_{1}\right) \times \hat{\mathcal{M}}_{J_{s}}\left(\varphi_{2}\right)\right|$, is even, unless $|k|>1$ and $\varphi=\varphi_{x, i, j}^{\alpha}$ or $\varphi=\varphi_{x, i, j}^{\beta}$ for some $(i, j)$, in which case it is odd. Therefore, for every $x \in \mathbb{T}_{\alpha} \cap \mathbb{T}_{\beta}$ and every $(i, j)$,

$\left(\partial \Psi_{i, j}+\Psi_{i, j} \partial\right)(x)$

$$
\begin{aligned}
& =\sum_{y \in \mathbb{T}_{\alpha} \cap \mathbb{T}_{\beta}} y \sum_{\substack{\varphi \in \pi_{2}(x, y) \\
\varphi \cdot Z=\varphi \cdot Z_{i, j}=1 \\
\mu(\varphi)=2}} \sum_{\begin{array}{c}
\mu\left(\varphi_{1}, \varphi_{2}\right. \\
\left(\varphi_{1}\right)=\mu\left(\varphi_{2}\right)=1 \\
\varphi=\varphi_{1} * \varphi_{2}
\end{array}}\left|\hat{\mathcal{M}}_{J_{s}}\left(\varphi_{1}\right) \times \hat{\mathcal{M}}_{J_{s}}\left(\varphi_{2}\right)\right| \prod_{l, j} U_{l, j}^{\varphi \cdot W_{l, j}} \\
& =\left(U_{i, a_{i}(j)}+U_{i, b_{i}(j)}\right) x
\end{aligned}
$$


and

$\left(\partial \Phi_{i, j}+\Phi_{i, j} \partial\right)(x)$

$=\sum_{y \in \mathbb{T}_{\alpha} \cap \mathbb{T}_{\beta}} y \sum_{\substack{\varphi \in \pi_{2}(x, y) \\ \varphi \cdot Z=0 \\ \mu(\varphi)=2}} \sum_{\substack{\varphi_{1}, \varphi_{2} \\ \mu\left(\varphi_{1}\right)=1 \\ \varphi=\varphi_{1} * \varphi_{2}}}\left(\varphi_{1}+\varphi_{2}\right) \cdot W_{i, j}\left|\hat{\mathcal{M}}_{J_{s}}\left(\varphi_{1}\right) \times \hat{\mathcal{M}}_{J_{s}}\left(\varphi_{2}\right)\right| U_{i, j}^{-1} \prod_{l, j} U_{l, j}^{\varphi \cdot W_{l, j}}$

$$
=\sum_{y \in \mathbb{T}_{\alpha} \cap \mathbb{T}_{\beta}} y \sum_{\substack{\varphi \in \pi_{2}(x, y) \\ \varphi \cdot Z=0 \\ \mu(\varphi)=2}}\left(\varphi \cdot W_{i, j}\right) U_{i, j}^{-1} \sum_{\substack{\varphi_{1}, \varphi_{2} \\ \mu\left(\varphi_{1}\right)=\mu\left(\varphi_{2}\right)=1 \\ \varphi=\varphi_{1} * \varphi_{2}}}\left|\hat{\mathcal{M}}_{J_{s}}\left(\varphi_{1}\right) \times \hat{\mathcal{M}}_{J_{s}}\left(\varphi_{2}\right)\right| \prod_{l, j} U_{l, j}^{\varphi \cdot W_{l, J}}
$$

$=0$.

This completes the proof.

For $1 \leq i \leq l$, let $\Psi_{i}=\sum_{j} \Psi_{i, j}$ and $\Phi_{i}=\Phi_{i, s_{i}}$.

Theorem 4.2 For every $i$, the two $U_{i, j}$-equivariant chain maps $\Psi_{i}$ and $\Phi_{i}$ induce link-invariant maps from $\operatorname{CFL}(Y, L, p)$ to $C F L(Y, L, p)\left[-1,-\delta_{1 i}, \ldots,-\delta_{l i}\right]$ and $C F L(Y, L, p)\left[1, \delta_{1 i}, \ldots, \delta_{l i}\right]$, respectively, in $N\left(K\left(\mathcal{A}_{l}\right)\right)$.

The proof is a consequence of the following lemma:

Lemma 4.3 Let $\mathcal{H}^{\prime}=\left(\Sigma^{\prime}, \alpha^{\prime}, \beta^{\prime}, z^{\prime}, w^{\prime}\right)$ be another Heegaard diagram for $(L, p)$ and let $J_{s}^{\prime}$ be a path of nearly symmetric, almost complex structures on $\operatorname{Sym}^{n^{\prime}}\left(\Sigma^{\prime}\right)$. If $\Psi_{i}^{\prime}$ and $\Phi_{i}^{\prime}$ denote the two chain maps on $C F_{\left(\mathcal{H}^{\prime}, J_{s}^{\prime}\right)}$ then, for all $i$, both the maps $F_{\left(\mathcal{H}, J_{s}\right),\left(\mathcal{H}^{\prime}, J_{s}^{\prime}\right)} \Psi_{i}+\Psi_{i}^{\prime} F_{\left(\mathcal{H}, J_{s}\right),\left(\mathcal{H}^{\prime}, J_{s}^{\prime}\right)}$ and $F_{\left(\mathcal{H}, J_{s}\right),\left(\mathcal{H}^{\prime}, J_{s}^{\prime}\right)} \Phi_{i}+\Phi_{i}^{\prime} F_{\left(\mathcal{H}, J_{s}\right),\left(\mathcal{H}^{\prime}, J_{s}^{\prime}\right)}$ are chain homotopic to zero, where the chain homotopies are also $U_{i}$-equivariant.

Proof Let us first clarify a notational convention that we will follow for the rest of the proof and, indeed, occasionally during the rest of the paper. If an object in the Heegaard diagram $\mathcal{H}$ is denoted by some symbol $S$, then the corresponding object in the Heegaard diagram $\mathcal{H}^{\prime}$ is denoted by $\boldsymbol{S}^{\prime}$. For example, the boundary map in $\mathcal{H}^{\prime}$ is denoted by $\partial^{\prime}$, the markings on the $i^{\text {th }}$ link component $L_{i}$ are $z_{i, 1}^{\prime}, \ldots, z_{i, k_{i}^{\prime}}^{\prime}$, $w_{i, 1}^{\prime}, \ldots, w_{i, k_{i}^{\prime}}^{\prime}$, and the basepoint $p_{i}$ is $w_{i, s_{i}^{\prime}}^{\prime}$.

Using [13, Proposition 7.1] and [7, Lemma 2.4], we can assume that we are in one of the following four cases:

Case $1 \mathcal{H}^{\prime}=\mathcal{H}$.

Following the proof of [13, Theorem 6.1], we assume that $\mathfrak{j}^{\prime}=\mathfrak{j}$ and we choose a path $J_{s, t}$ in the space of paths of nearly symmetric, almost complex structures 
joining $J_{s}$ to $J_{s}^{\prime}$. Given a Whitney disk $\varphi \in \pi_{2}(x, y)$, let $\mathcal{M}_{J_{s, t}}(\varphi)$ denote the moduli space of holomorphic disks with time-dependent complex structure on the target [13, Equation (14)]. The map $F_{\left(\mathcal{H}, J_{s}\right),\left(\mathcal{H}, J_{s}^{\prime}\right)}$ is defined as

$$
F_{\left(\mathcal{H}, J_{s}\right),\left(\mathcal{H}, J_{s}^{\prime}\right)}(x)=\sum_{y \in \mathbb{T}_{\alpha} \cap \mathbb{T}_{\beta}} y \sum_{\substack{\varphi \in \pi_{2}(x, y) \\ \varphi \cdot Z=0 \\ \mu(\varphi)=0}}\left|\mathcal{M}_{J_{s, t}}(\varphi)\right| \prod_{l, j} U_{l, j}^{\varphi \cdot W_{l, J}} .
$$

Define $H_{z_{i}}, H_{w_{i}}: C F_{\left(\mathcal{H}, J_{s}\right)} \rightarrow C F_{\left(\mathcal{H}, J_{s}^{\prime}\right)}^{3}$ as follows:

$$
\begin{aligned}
H_{z_{i}}(x) & =\sum_{y \in \mathbb{T}_{\alpha} \cap \mathbb{T}_{\beta}} y \sum_{\substack{\varphi \in \pi_{2}(x, y) \\
\varphi \cdot Z=\varphi \cdot Z_{i}=1 \\
\mu(\varphi)=0}}\left|\mathcal{M}_{J_{s, t}}(\varphi)\right| \prod_{l, j} U_{l, j}^{\varphi \cdot W_{l, j}}, \\
H_{w_{i}}(x)= & \sum_{y \in \mathbb{T}_{\alpha} \cap \mathbb{T}_{\beta} \beta} y \sum_{\substack{\varphi \in \pi_{2}(x, y) \\
\varphi \cdot Z=0 \\
\mu(\varphi)=0}} \varphi \cdot W_{i, s_{i}}\left|\mathcal{M}_{J_{s, t}}(\varphi)\right| U_{i, s_{i}}^{-1} \prod_{l, j} U_{l, j}^{\varphi \cdot W_{l, j}} .
\end{aligned}
$$

By analyzing the ends of $\mathcal{M}_{J_{s, t}}(\varphi)$ for Whitney disks $\varphi$ with $\mu(\varphi)=1$, we see that

$$
\begin{aligned}
& F_{\left(\mathcal{H}, J_{s}\right),\left(\mathcal{H}^{\prime}, J_{s}^{\prime}\right)} \Psi_{i}+\Psi_{i}^{\prime} F_{\left(\mathcal{H}, J_{s}\right),\left(\mathcal{H}^{\prime}, J_{s}^{\prime}\right)}=H_{z_{i}} \partial+\partial^{\prime} H_{z_{i}}, \\
& F_{\left(\mathcal{H}, J_{s}\right),\left(\mathcal{H}^{\prime}, J_{s}^{\prime}\right)} \Phi_{i}+\Phi_{i}^{\prime} F_{\left(\mathcal{H}, J_{s}\right),\left(\mathcal{H}^{\prime}, J_{s}^{\prime}\right)}=H_{w_{i}} \partial+\partial^{\prime} H_{w_{i}} .
\end{aligned}
$$

Case $2 J_{s}^{\prime}=J_{s}$ and $\mathcal{H}^{\prime}$ can be obtained from $\mathcal{H}$ by isotoping and handlesliding the $\alpha$ curves or by isotoping and handlesliding the $\beta$ curves.

Without loss of generality, let us assume that we are isotoping and handlesliding the $\beta$ curves. Furthermore, we can assume that the multicurves $\beta$ and $\beta^{\prime}$ intersect each other transversely, $\widetilde{\mathcal{H}}=\left(\Sigma, \beta, \beta^{\prime}, z, w\right)$ is an admissible Heegaard diagram for the $n$-component unlink in $\#^{g}\left(S^{1} \times S^{2}\right)$ and the two tori $\mathbb{T}_{\beta}$ and $\mathbb{T}_{\beta^{\prime}}$ intersect each other at $2^{n}$ points, all lying in the same Alexander grading.

In $\widetilde{\mathcal{H}}$ let $\Theta \in \mathbb{T}_{\beta} \cap \mathbb{T}_{\beta^{\prime}}$ be the element with the highest Maslov grading. By [13, Lemma 9.1 and Lemma 9.4], $\Theta$ is a cycle in $C F_{\left(\widetilde{\mathcal{H}}, J_{s}\right)}$. The map $F_{\left(\mathcal{H}, J_{s}\right),\left(\mathcal{H}^{\prime}, J_{s}\right)}$, evaluated on $x \in \mathbb{T}_{\alpha} \cap \mathbb{T}_{\beta}$, is given by

$$
F_{\left(\mathcal{H}, J_{s}\right),\left(\mathcal{H}^{\prime}, J_{s}\right)}(x)=\sum_{y \in \mathbb{T}_{\alpha} \cap \mathbb{T}_{\beta^{\prime}}} y \sum_{\substack{\varphi \in \pi_{2}(x, \Theta, y) \\ \varphi \cdot Z=0 \\ \mu(\varphi)=0}}\left|\mathcal{M}_{J_{s}}(\varphi)\right| \prod_{l, j} U_{l, j}^{\varphi \cdot W_{l, J}},
$$

${ }^{3}$ Technically some shift operators are involved: $H_{z_{i}}$ maps $C F_{\left(\mathcal{H}, J_{S}\right)}$ to $C F_{\left(\mathcal{H}, J_{s}^{\prime}\right)}\left[0,-\delta_{1 i}, \ldots,-\delta_{l i}\right]$ and $H_{w_{i}}$ maps $C F_{\left(\mathcal{H}, J_{s}\right)}$ to $C F_{\left(\mathcal{H}, J_{s}^{\prime}\right)}\left[2, \delta_{1 i}, \ldots, \delta_{l i}\right]$. However, we will often suppress the degree shift information. 


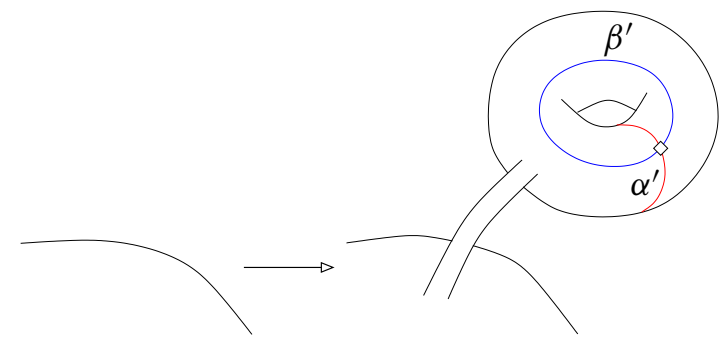

Figure 4.1: An ordinary stabilization

where $\pi_{2}(x, \Theta, y)$ is the set of all Whitney triangles connecting $x, \Theta$ and $y$ [13, Section 8.1.2]. Define $H_{z_{i}}, H_{w_{i}}: C F_{\left(\mathcal{H}, J_{s}\right)} \rightarrow C F_{\left(\mathcal{H}^{\prime}, J_{s}\right)}$ as follows:

$$
\begin{aligned}
& H_{z_{i}}(x)=\sum_{y \in \mathbb{T}_{\alpha} \cap \mathbb{T}_{\beta^{\prime}}} y \sum_{\substack{\varphi \in \pi_{2}(x, \Theta, y) \\
\varphi \cdot Z=\varphi \cdot Z_{i}=1 \\
\mu(\varphi)=0}}\left|\mathcal{M}_{J_{s}}(\varphi)\right| \prod_{l, j} U_{l, j}^{\varphi \cdot W_{l, J}}, \\
& H_{w_{i}}(x)=\sum_{y \in \mathbb{T}_{\alpha} \cap \mathbb{T}_{\beta^{\prime}}} y \sum_{\substack{\varphi \in \pi_{2}(x, \Theta, y) \\
\varphi \cdot Z=0 \\
\mu(\varphi)=0}} \varphi \cdot W_{i, s_{i}}\left|\mathcal{M}_{J_{s}}(\varphi)\right| U_{i, s_{i}}^{-1} \prod_{l, j} U_{l, j}^{\varphi \cdot W_{l, J}} .
\end{aligned}
$$

Since $\Theta$ is a cycle in $C F_{\left(\widetilde{\mathcal{H}}, J_{s}\right)}$ and all the points in $\mathbb{T}_{\beta} \cap \mathbb{T}_{\beta^{\prime}}$ lie in the same Alexander grading, by counting the ends of $\mathcal{M}_{J_{s}}(\varphi)$ for Whitney triangles $\varphi$ with $\mu(\varphi)=1$ we get

$$
\begin{aligned}
& F_{\left(\mathcal{H}, J_{s}\right),\left(\mathcal{H}^{\prime}, J_{s}^{\prime}\right)} \Psi_{i}+\Psi_{i}^{\prime} F_{\left(\mathcal{H}, J_{s}\right),\left(\mathcal{H}^{\prime}, J_{s}^{\prime}\right)}=H_{z_{i}} \partial+\partial^{\prime} H_{z_{i}}, \\
& F_{\left(\mathcal{H}, J_{s}\right),\left(\mathcal{H}^{\prime}, J_{s}^{\prime}\right)} \Phi_{i}+\Phi_{i}^{\prime} F_{\left(\mathcal{H}, J_{s}\right),\left(\mathcal{H}^{\prime}, J_{s}^{\prime}\right)}=H_{w_{i}} \partial+\partial^{\prime} H_{w_{i}} .
\end{aligned}
$$

Case $3 \mathcal{H}^{\prime}$ is obtained from $\mathcal{H}$ by an ordinary (de)stabilization, as shown in Figure 4.1, and $J_{S}^{\prime}$ is related to $J_{S}$ as described below.

We fix a Riemann surface $E$ of genus 1 with one $\alpha^{\prime}$-circle and one $\beta^{\prime}$-circle, intersecting each other transversely at a single point. The Heegaard surface $\Sigma^{\prime}$ is simply $\Sigma \# E$; $J_{S}^{\prime}$ is induced from $J_{S}$, the complex structure on $E$, the two connected sum points in $\Sigma$ and $E$, and the length of the connected sum neck.

There is a natural bijection between $\mathbb{T}_{\alpha} \cap \mathbb{T}_{\beta}$ and $\mathbb{T}_{\alpha^{\prime}} \cap \mathbb{T}_{\beta^{\prime}}$; let $x^{\prime} \in \mathbb{T}_{\alpha^{\prime}} \cap \mathbb{T}_{\beta^{\prime}}$ be the element corresponding to $x \in \mathbb{T}_{\alpha} \cap \mathbb{T}_{\beta}$. For all $x, y \in \mathbb{T}_{\alpha} \cap \mathbb{T}_{\beta}$, there is a natural bijection between $\pi_{2}(x, y)$ and $\pi_{2}\left(x^{\prime}, y^{\prime}\right)$ which preserves the Maslov index; let $\varphi^{\prime} \in \pi_{2}\left(x^{\prime}, y^{\prime}\right)$ be the Whitney disk corresponding to $\varphi \in \pi_{2}(x, y)$.

However, by moving the connected sum points and extending the connected sum length, we can ensure that, for all $x, y \in \mathbb{T}_{\alpha} \cap \mathbb{T}_{\beta}$ and all $\varphi \in \pi_{2}(x, y)$ with $\mu(\varphi)=1$, the 


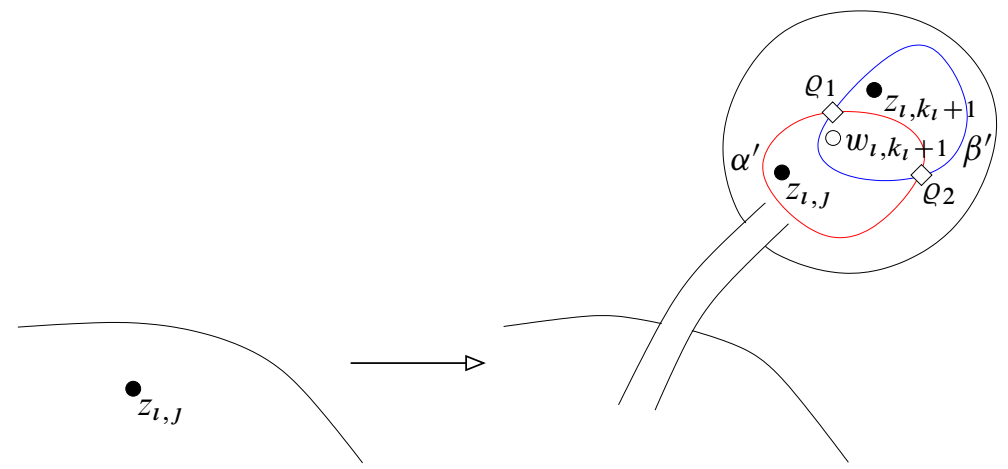

Figure 4.2: A special stabilization

two moduli spaces $\hat{\mathcal{M}}_{J_{s}}(\varphi)$ and $\hat{\mathcal{M}}_{J_{s}^{\prime}}\left(\varphi^{\prime}\right)$ are homeomorphic [13, Theorem 10.4]. The map $F_{\left(\mathcal{H}, J_{s}\right),\left(\mathcal{H}^{\prime}, J_{s}^{\prime}\right)}$ sends $x$ to $x^{\prime}$ and the map $F_{\left(\mathcal{H}^{\prime}, J_{s}^{\prime}\right),\left(\mathcal{H}, J_{s}\right)}$ is its inverse. Therefore, $F_{\left(\mathcal{H}, J_{s}\right),\left(\mathcal{H}^{\prime}, J_{s}^{\prime}\right)} \Psi_{i}+\Psi_{i}^{\prime} F_{\left(\mathcal{H}, J_{s}\right),\left(\mathcal{H}^{\prime}, J_{s}^{\prime}\right)}=F_{\left(\mathcal{H}, J_{s}\right),\left(\mathcal{H}^{\prime}, J_{s}^{\prime}\right)} \Phi_{i}+\Phi_{i}^{\prime} F_{\left(\mathcal{H}, J_{s}\right),\left(\mathcal{H}^{\prime}, J_{s}^{\prime}\right)}=0$ and $F_{\left(\mathcal{H}^{\prime}, J_{s}^{\prime}\right),\left(\mathcal{H}, J_{s}\right)} \Psi_{i}^{\prime}+\Psi_{i} F_{\left(\mathcal{H}^{\prime}, J_{s}^{\prime}\right),\left(\mathcal{H}, J_{s}\right)}=F_{\left(\mathcal{H}^{\prime}, J_{s}^{\prime}\right),\left(\mathcal{H}, J_{s}\right)} \Phi_{i}^{\prime}+\Phi_{i} F_{\left(\mathcal{H}^{\prime}, J_{s}^{\prime}\right),\left(\mathcal{H}, J_{s}\right)}=0$.

Case $4 \mathcal{H}^{\prime}$ is obtained from $\mathcal{H}$ by a special (de)stabilization, as shown in Figure 4.2, and $J_{s}^{\prime}$ is related to $J_{s}$ as described below.

We fix a Riemann surface $S$ of genus 0 with one $\alpha^{\prime}$-circle and one $\beta^{\prime}$-circle, intersecting each other transversely at two points $\varrho_{1}$ and $\varrho_{2}$. The Heegaard surface $\Sigma^{\prime}$ is simply $\Sigma \# S$, where the connected sum is done near the $z_{l, j}$ marking on $\Sigma$ and the $z$ and $w$ markings on $\Sigma^{\prime}$ are as shown in Figure 4.2; $J_{s}^{\prime}$ is induced from $J_{s}$, the complex structure on $S$, the two connected sum points in $\Sigma$ and $S$, and the length of the connected sum neck.

There is a natural bijection between $\mathbb{T}_{\alpha^{\prime}} \cap \mathbb{T}_{\beta^{\prime}}$ and $\left(\mathbb{T}_{\alpha} \cap \mathbb{T}_{\beta}\right) \times\{1,2\}$, where the element corresponding to $(x, 1)$ uses the point $\varrho_{1}$ and is denoted by $x^{\prime}$ and the element corresponding to $(x, 2)$ uses the point $\varrho_{2}$ and is denoted by $x^{\prime \prime}$. Recall that $C F_{\left(\mathcal{H} . J_{s}\right)}$ is the module generated freely over $\mathbb{P}$ by $\mathbb{T}_{\alpha} \cap \mathbb{T}_{\beta}$ and $C F_{\left(\mathcal{H}^{\prime}, J_{s}^{\prime}\right)}$ is the module generated freely over $\mathbb{P}^{\prime}=\mathbb{P} \otimes \mathbb{F}_{2}\left[U_{l, k_{l}+1}\right]$ by $\mathbb{T}_{\alpha^{\prime}} \cap \mathbb{T}_{\beta^{\prime}}$. All the maps below are assumed to be $\mathbb{P}$-module maps; furthermore, the maps between $\mathbb{P}^{\prime}$ modules are assumed to be $\mathbb{P}^{\prime}$-module maps. From [15, Proposition 6.5], by moving the connected sum point in $S$ near the $\alpha^{\prime}$-circle and extending the connected sum length, we can ensure the following: $C F_{\left(\mathcal{H}^{\prime}, J_{s}^{\prime}\right)}$ is isomorphic to the mapping cone $U_{l, k_{l}+1}+U_{l, a_{l}(J)}: C_{2} \otimes \mathbb{F}_{2}\left[U_{l, k_{l}+1}\right] \rightarrow C_{1} \otimes \mathbb{F}_{2}\left[U_{l, k_{l}+1}\right]$, where

- $C_{1}$ is isomorphic to $C F_{\left(\mathcal{H}, J_{S}\right)}$, corresponding to elements of the form $x^{\prime}$, and $C_{2}$ is isomorphic to $C F_{\left(\mathcal{H}, J_{s}\right)}$, corresponding to elements of the form $x^{\prime \prime}$;

- $F_{\left(\mathcal{H}, J_{s}\right),\left(\mathcal{H}^{\prime}, J_{s}^{\prime}\right)}(x)=x^{\prime}$; 
- $F_{\left(\mathcal{H}^{\prime}, J_{s}^{\prime}\right),\left(\mathcal{H}, J_{s}\right)}\left(U_{l, k_{l}+1}^{m} x^{\prime \prime}\right)=0$ and $F_{\left(\mathcal{H}^{\prime}, J_{s}^{\prime}\right),\left(\mathcal{H}, J_{s}\right)}\left(U_{l, k_{l}+1}^{m} x^{\prime}\right)=U_{l, a_{l}(j)}^{m} x$;

- $\Psi_{i, j}^{\prime}\left(x^{\prime}\right)=\left(\Psi_{i, j}(x)\right)^{\prime}$ and $\Psi_{i, j}^{\prime}\left(x^{\prime \prime}\right)=\left(\Psi_{i, j}(x)\right)^{\prime \prime}$ for $(i, j) \notin\left\{(l, j),\left(l, k_{l}+1\right)\right\}$;

- $\left(\Psi_{l, j}^{\prime}+\Psi_{l, k_{l}+1}^{\prime}\right)\left(x^{\prime}\right)=\left(\Psi_{l, j}(x)\right)^{\prime}$ and $\left(\Psi_{l, j}^{\prime}+\Psi_{l, k_{l}+1}^{\prime}\right)\left(x^{\prime \prime}\right)=\left(\Psi_{l, j}(x)\right)^{\prime \prime}$;

- $\Phi_{i}^{\prime}\left(x^{\prime}\right)=\left(\Phi_{i}(x)\right)^{\prime}$ and $\Phi_{i}^{\prime}\left(x^{\prime \prime}\right)=\left(\Phi_{i}(x)\right)^{\prime \prime}+x^{\prime} \delta_{i l} \delta_{s_{i} a_{l}(\jmath)}$.

Therefore,

$$
F_{\left(\mathcal{H}, J_{s}\right),\left(\mathcal{H}^{\prime}, J_{s}^{\prime}\right)} \Psi_{i}+\Psi_{i}^{\prime} F_{\left(\mathcal{H}, J_{s}\right),\left(\mathcal{H}^{\prime}, J_{s}^{\prime}\right)}=F_{\left(\mathcal{H}, J_{s}\right),\left(\mathcal{H}^{\prime}, J_{s}^{\prime}\right)} \Phi_{i}+\Phi_{i}^{\prime} F_{\left(\mathcal{H}, J_{s}\right),\left(\mathcal{H}^{\prime}, J_{s}^{\prime}\right)}=0 .
$$

Similarly, we get

$$
\begin{aligned}
F_{\left(\mathcal{H}^{\prime}, J_{s}^{\prime}\right),\left(\mathcal{H}, J_{s}\right)} \Psi_{i}^{\prime}+\Psi_{i} F_{\left(\mathcal{H}^{\prime}, J_{s}^{\prime}\right),\left(\mathcal{H}, J_{s}\right)} & =0 \\
\left(F_{\left(\mathcal{H}^{\prime}, J_{s}^{\prime}\right),\left(\mathcal{H}, J_{s}\right)} \Phi_{i}^{\prime}+\Phi_{i} F_{\left(\mathcal{H}^{\prime}, J_{s}^{\prime}\right),\left(\mathcal{H}, J_{s}\right)}\right)\left(U_{l, k_{l}+1}^{m} x^{\prime}\right) & =0 .
\end{aligned}
$$

However,

$$
\left(F_{\left(\mathcal{H}^{\prime}, J_{s}^{\prime}\right),\left(\mathcal{H}, J_{s}\right)} \Phi_{i}^{\prime}+\Phi_{i} F_{\left(\mathcal{H}^{\prime}, J_{s}^{\prime}\right),\left(\mathcal{H}, J_{s}\right)}\right)\left(U_{l, k_{l}+1}^{m} x^{\prime \prime}\right)=U_{l, a_{l}(j)}^{m} x \delta_{i l} \delta_{s_{i} a_{l}(\jmath)} .
$$

Define $H_{w_{i}}: C F_{\left(\mathcal{H}^{\prime}, J_{s}^{\prime}\right)} \rightarrow C F_{\left(\mathcal{H}, J_{s}\right)}$ by $H_{w_{i}}\left(U_{l, k_{l}+1}^{m} x^{\prime \prime}\right)=0$ and $H_{w_{i}}\left(U_{l, k_{l}+1}^{m} x^{\prime}\right)=$ $m U_{l, a_{l}(J)}^{m-1} x \delta_{i l} \delta_{s_{i} a_{l}(J)}$. A careful analysis shows that

$$
F_{\left(\mathcal{H}^{\prime}, J_{s}^{\prime}\right),\left(\mathcal{H}, J_{s}\right)} \Phi_{i}^{\prime}+\Phi_{i} F_{\left(\mathcal{H}^{\prime}, J_{s}^{\prime}\right),\left(\mathcal{H}, J_{s}\right)}=H_{w_{i}} \partial^{\prime}+\partial H_{w_{i}}
$$

Observe that the chain homotopy is still $U_{i}$-equivariant.

We will now state and prove some properties of the maps $\Psi_{i}$ and $\Phi_{i}$.

Lemma 4.4 For all $i$, all three of the maps $\Psi_{i} \Phi_{i}+\Phi_{i} \Psi_{i}, \Psi_{i}^{2}$ and $\Phi_{i}^{2}$ are chain homotopic to zero, and the chain homotopies are also $U_{i, j}$-equivariant.

Proof Define the three $U_{i, j}$-equivariant chain homotopies as follows:

$$
\begin{aligned}
& H_{1, i}(x)=\sum_{y \in \mathbb{T}_{\alpha} \cap \mathbb{T}_{\beta}} y \sum_{\substack{\varphi \in \pi_{2}(x, y) \\
\varphi \cdot Z=\varphi \cdot Z_{i}=1 \\
\mu(\varphi)=1}}\left(\varphi \cdot W_{i, s_{i}}\right)\left|\hat{\mathcal{M}}_{J_{s}}(\varphi)\right| U_{i, s_{i}}^{-1} \prod_{l, j} U_{l, j}^{\varphi \cdot W_{l, j}}, \\
& H_{2, i}(x)=\sum_{y \in \mathbb{T}_{\alpha} \cap \mathbb{T}_{\beta}} y \sum_{\substack{\varphi \in \pi_{2}(x, y) \\
\varphi \cdot Z=\varphi \cdot Z_{i}=2 \\
\mu(\varphi)=1}}\left|\hat{\mathcal{M}}_{J_{s}}(\varphi)\right| \prod_{l, j} U_{l, j}^{\varphi \cdot W_{l, j}}, \\
& H_{3, i}(x)=\sum_{y \in \mathbb{T}_{\alpha} \cap \mathbb{T}_{\beta}} y \sum_{\substack{\varphi \in \pi_{2}(x, y) \\
\varphi \cdot Z=0 \\
\mu(\varphi)=1}}\left(\begin{array}{c}
W_{i, s_{i}} \\
2
\end{array}\right)\left|\hat{\mathcal{M}}_{J_{s}}(\varphi)\right| U_{i, s_{i}}^{-2} \prod_{l, j} U_{l, j}^{\varphi \cdot W_{l, j}} .
\end{aligned}
$$


By counting the ends of $\hat{\mathcal{M}}_{J_{s}}(\varphi)$ for Whitney disks $\varphi$ with $\mu(\varphi)=2$ and $\varphi \cdot Z=$ $\varphi \cdot Z_{i}=1$, we see that $\Psi_{i} \Phi_{i}+\Phi_{i} \Psi_{i}=\partial H_{1, i}+H_{1, i} \partial$. When $\varphi \cdot W_{i}=1$, we might have boundary degenerations in the ends of $\hat{\mathcal{M}}_{J_{s}}(\varphi)$; however, they cancel in pairs [15, Theorem 5.5]. Similarly, by counting the ends of $\hat{\mathcal{M}}_{J_{s}}(\varphi)$ for Whitney disks $\varphi$ with $\mu(\varphi)=2$ and $\varphi \cdot Z=\varphi \cdot Z_{i}=2$, we see that $\Psi_{i}^{2}=\partial H_{2, i}+H_{2, i} \partial$. For the third case, let us explicitly do the calculation:

$$
\begin{aligned}
& \left(\left[H_{3, i}: \partial\right]+\Phi_{i}^{2}\right)(x) \\
& \begin{aligned}
=\sum_{y \in \mathbb{T}_{\alpha} \cap \mathbb{T}_{\beta}} y \sum_{\substack{\varphi \in \pi_{2}(x, y) \\
\varphi \cdot Z=0 \\
\mu(\varphi)=2}} \sum_{\begin{array}{c}
\varphi_{1}, \varphi_{2}, m_{1}, m_{2} \\
\varphi_{1} \cdot W_{i, s_{i}}=m_{1} \\
\varphi_{2} \cdot W_{i, s_{i}}=m_{2} \\
\mu\left(\varphi_{1}\right)=1 \\
\varphi=\varphi_{1} * \varphi_{2}
\end{array}}\left[\left(\left(\begin{array}{c}
m_{1} \\
2
\end{array}\right)+\left(\begin{array}{c}
m_{2} \\
2
\end{array}\right)+m_{1} m_{2}\right)\right. \\
\left.\mu\left|\hat{\mathcal{M}}_{J_{s}}\left(\varphi_{1}\right) \times \hat{\mathcal{M}}_{J_{s}}\left(\varphi_{2}\right)\right| U_{i, s_{i}}^{-2} \prod_{l, j} U_{l, J}^{\varphi \cdot W_{l, J}}\right]
\end{aligned} \\
& =\sum_{y \in \mathbb{T}_{\alpha} \cap \mathbb{T}_{\beta}} y \sum_{\substack{\varphi \in \pi_{2}(x, y) \\
\varphi \cdot Z=0}} \sum_{\begin{array}{c}
\varphi_{1}, \varphi_{2}, m_{1}, m_{2} \\
\varphi^{\prime} \cdot W_{i, s_{i}}=m_{1} \\
\varphi_{2} \cdot W_{i, s_{i}}=m_{2} \\
\mu\left(\varphi_{1}\right)=1 \\
\varphi=\varphi_{1} * \varphi_{2}
\end{array}}\left(\begin{array}{c}
m_{1}+m_{2} \\
2
\end{array}\right)\left|\hat{\mathcal{M}}_{J_{s}}\left(\varphi_{1}\right) \times \hat{\mathcal{M}}_{J_{s}}\left(\varphi_{2}\right)\right| U_{i, s_{i}}^{-2} \prod_{l, j} U_{l, j}^{\varphi \cdot W_{l, J}} \\
& =\sum_{y \in \mathbb{T}_{\alpha} \cap \mathbb{T}_{\beta}} y \sum_{\substack{\varphi \in \pi_{2}(x, y) \\
\varphi \cdot Z=0 \\
\mu(\varphi)=2}}\left(\begin{array}{c}
\varphi \cdot W_{i, s_{i}} \\
2
\end{array}\right) U_{i, s_{i}}^{-2} \sum_{\substack{\varphi_{1}, \varphi_{2} \\
\mu\left(\varphi_{1}\right)=1 \\
\varphi=\varphi_{1} * \varphi_{2}}}\left|\hat{\mathcal{M}}_{J_{s}}\left(\varphi_{1}\right) \times \hat{\mathcal{M}}_{J_{s}}\left(\varphi_{2}\right)\right| \prod_{l, j} U_{l, j}^{\varphi \cdot W_{l, j}} \\
& =0 \text {. }
\end{aligned}
$$

This completes the proof.

Theorem 4.5 For all $i$, the map $\mathrm{Id}+\Psi_{i} \Phi_{i}=\mathrm{Id}+\Phi_{i} \Psi_{i}$ is a link-invariant involution of $C F L(Y, L, p)$, viewed as an object in $N\left(K\left(\mathcal{A}_{l}\right)\right)$.

Proof We already know from Theorem 4.2 that $\mathrm{Id}+\Psi_{i} \Phi_{i}$ is a link-invariant map from $C F L(Y, L, p)$ to itself. To see that it is an involution, observe that in $N\left(K\left(\mathcal{A}_{l}\right)\right)$, by Lemma 4.4, $\left(\mathrm{Id}+\Psi_{i} \Phi_{i}\right)^{2}=\mathrm{Id}+\Psi_{i} \Phi_{i} \Psi_{i} \Phi_{i}=\mathrm{Id}+\Psi_{i} \Psi_{i} \Phi_{i} \Phi_{i}=\mathrm{Id}$.

Instead of working in the slightly unfamiliar category $N\left(K\left(\mathcal{A}_{l}\right)\right)$, we often take the homology and work with $\operatorname{HFL}(Y, L, p)=H_{*}(C F L(Y, L, p))$ in $N\left(\mathcal{B}_{l}\right)$. Another standard object to work with is the hat-invariant $\widehat{H F} L(Y, L, p)$ living in $N\left(\mathcal{C}_{l}\right)$ : it is the homology of the mapping cone of all the maps $U_{1}, \ldots, U_{l}$ in $\operatorname{CFL}(Y, L, p)$.

The map Id $+\Psi_{i} \Phi_{i}$ induces link-invariant involutions on the objects $\operatorname{HFL}(Y, L, p)$ and $\widehat{H F} L(Y, L, p)$. Even though the involution on $\widehat{H F} L(Y, L, p)$ (and hence the one 
on $\operatorname{CFL}(Y, L, p))$ is often non-trivial - see Theorem 6.1 - quite (un-)surprisingly, the involution on $\operatorname{HFL}(Y, L, p)$ is always the identity.

Lemma 4.6 For all $i$, the involution $\operatorname{Id}+\Psi_{i} \Phi_{i}$ on $\operatorname{HFL}(Y, L, p)$, viewed as an object in $N\left(\mathcal{B}_{l}\right)$, is the identity map.

Proof In order to prove this, we only need to show that the map $\Psi_{i} \Phi_{i}$ is chain homotopic to zero, where the chain homotopy need not be $U_{i}$-equivariant. Fix a Heegaard diagram $\mathcal{H}$ and a path of nearly symmetric, almost complex structures $J_{S}$ on the symmetric product. The chain homotopy $H_{i}$ is $U_{l, j}$-equivariant for all $(l, J) \neq\left(i, s_{i}\right)$, and is defined as follows:

$$
H_{i}\left(U_{i, s_{i}}^{m} x\right)=\sum_{y \in \mathbb{T}_{\alpha} \cap \mathbb{T}_{\beta}} y \sum_{\substack{\varphi \in \pi_{2}(x, y) \\ \varphi \cdot Z=\varphi \cdot Z_{i}=1 \\ \mu(\varphi)=1}} m\left|\hat{\mathcal{M}}_{J_{s}}(\varphi)\right| U_{i, s_{i}}^{m-1} \prod_{l, j} U_{l, j}^{\varphi \cdot W_{l, J}}
$$

A careful analysis of the ends of $\hat{\mathcal{M}}_{J_{s}}(\varphi)$ for Whitney disks $\varphi$ with $\mu(\varphi)=2$ and $\varphi \cdot Z=\varphi \cdot Z_{i}=1$ shows that $\Psi_{i} \Phi_{i}=\partial H_{i}+H_{i} \partial$.

In view of Lemma 4.6 along with Theorem 1.1, we can define the invariant $H F L$ for unpointed links in $S^{3}$, although, due to Theorem 6.1, we must continue to treat $C F L$ and $\widehat{H F} L$ as invariants of pointed links. Let us conclude this section with a rather bold conjecture, which is somewhat justified by Theorem 1.1.

Conjecture 4.7 The two automorphisms $\rho\left(\sigma_{i}\right)$ and $\mathrm{Id}+\Psi_{i} \Phi_{i}$ are equal in the cate-

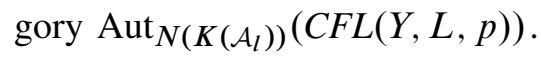

\section{Grid diagrams}

In this section, we concentrate on pointed links in $S^{3}$. The main aim is to prove Theorem 1.1. We focus our attention on the component $\left(L_{1}, p_{1}\right)$ of the $l$-component pointed link $(L, p)$ in $S^{3}$. Following [7], we will represent such links by a special type of Heegaard diagrams called grid diagrams. A grid diagram of index $n$ is a Heegaard diagram $\mathcal{G}=(T, \alpha, \beta, z, w)$ for $(L, p)$, where the Heegaard surface $T$ is the torus obtained as a quotient of $[0,1]^{2} \subset \mathbb{C}$ by identifying opposite sides, $\alpha$ is a multicurve which is isotopic to the image of $[0,1] \times\{0,1 / n, \ldots,(n-1) / n\}, \beta$ is a multicurve which is isotopic to the image of $\{0,1 / n, \ldots,(n-1) / n\} \times[0,1]$, and each $\alpha$-circle intersects each $\beta$-circle at exactly one point. It is easy to see that each pointed link can be represented by a grid diagram. We usually take $J_{S}$ to be the constant path of 
the product complex structure on $\operatorname{Sym}^{n}(T)$ induced from the complex structure on $\mathbb{C}$. By generically perturbing $\alpha$ and $\beta$, we can ensure that $J_{s}$ achieves transversality [4, Proposition 3.9]. We will keep using our notations from the previous sections. However, to avoid clutter, from now on, unless we deem it to be particularly illuminating, we will drop the subscript $J_{s}$ from our notation. We sometimes use the words north, south, east and west to denote local directions on the torus $T$ (ie directions on some contractible subset of $T$ ); at all such times, it is implicitly understood that we have isotoped the $\alpha-$ and $\beta$-circles to horizontal and vertical circles, respectively.

We will set up for the proof, while doing a brief review of grid diagrams, in the following few subsections.

\subsection{The grid chain complex}

Given a grid diagram $\mathcal{G}$ of index $n$, there are exactly $n$ ! points in $\mathbb{T}_{\alpha} \cap \mathbb{T}_{\beta}$. If $\varphi \in \pi_{2}(x, y)$ for some $x, y \in \mathbb{T}_{\alpha} \cap \mathbb{T}_{\beta}$, then let $D(\varphi)$ be the shadow of $\varphi$, which is a 2-chain generated by components of $T \backslash(\alpha \cup \beta)$ [13, Definition 2.13]. The domain $D(\varphi)$ is said to be non-negative if it has non-negative coefficients everywhere. Since we are working with a product complex structure, if $\mathcal{M}_{J_{s}}(\varphi) \neq \varnothing$, then $D(\varphi)$ is non-negative. The central fact about grid diagrams is the following observation:

Theorem 5.1 [7] If $\varphi \in \pi_{2}(x, y)$ is a Whitney disk in a grid diagram with $D(\varphi)$ non-negative, then $\mu(\varphi) \geq 0$. Furthermore, $\mu(\varphi)=0$ happens precisely when $x=y$ and $\varphi$ is the trivial disk, and $\mu(\varphi)=1$ happens precisely when $x$ and $y$ differ in exactly two coordinates, $D(\varphi)$ is a properly embedded rectangle in $(T, \alpha \cup \beta)$ which does not contain any coordinates of $x$ or $y$ in its interior, the northeast and southwest corners of $D(\varphi)$ are coordinates of $x$, and the northwest and southeast corners of $D(\varphi)$ are coordinates of $y$; in the latter case, $\hat{\mathcal{M}}(\varphi)$ has exactly one point.

In particular, this theorem implies that the grid chain complex $C F_{\left(\mathcal{G}, J_{s}\right)} \in \mathrm{Ob}_{K\left(\mathcal{A}_{l}\right)}$ is independent of $J_{S}$ as long as it is a constant path of the product complex structure induced from some complex structure on $T$. In fact, the following is an explicit description of the chain complex $C F_{\mathcal{G}}$ in grid diagram terminology.

A state $x$ is an $n$-tuple of points $x=\left(x_{1}, \ldots, x_{n}\right)$ (and the points $x_{i}$ are called the coordinates of $x$ ) such that each $\alpha$-circle contains some $x_{i}$ and each $\beta$-circle contains some $x_{j}$. Clearly there are $n$ ! states, and there is a natural bijection between $\mathbb{T}_{\alpha} \cap \mathbb{T}_{\beta}$ and the set of all states $\mathcal{S}_{\mathcal{G}}$. A grid 2-chain is a formal linear combination of the $n^{2}$ components of $T \backslash(\alpha \cup \beta)$ over $\mathbb{Z}$. A grid 2-chain is said to be positive if all its coefficients are non-negative. Given a point $p \in T \backslash(\alpha \cup \beta)$ and a grid 2-chain $D$, the 
number $n_{p}(D)$ is the coefficient of $D$ at the component of $T \backslash(\alpha \cup \beta)$ that contains the point $p$. For any grid 2-chain $D$, let $n_{z_{i}}(D)=\sum_{j} n_{z_{i, j}}(D), n_{w_{i}}(D)=\sum_{j} n_{w_{i, j}}(D)$, $n_{z}(D)=\sum_{i} n_{z_{i}}(D)$ and $n_{w}(D)=\sum_{i} n_{w_{i}}(D)$. A domain joining a state $x$ to a state $y$ is a grid 2-chain $D$ such that $\partial(\partial D \cap \alpha)=y-x$. The set of all domains joining $x$ to $y$ is denoted by $\mathcal{D}_{\mathcal{G}}(x, y)$ and it is in natural bijection with $\pi_{2}(x, y)$. A rectangle $R \in \mathcal{D}_{\mathcal{G}}(x, y)$ is a domain satisfying the following conditions: each coefficient of $R$ is either 0 or 1 ; the closure of the region where $R$ has coefficient 1 is properly embedded rectangle in $T$; that rectangle does not contain any coordinates of $x$ or $y$ in its interior; and the northeast and southwest corners of that rectangle are coordinates of $x$ and the northwest and southeast corners of that rectangle are coordinates of $y$. The set of all rectangles joining $x$ to $y$ is denoted by $\mathcal{R}_{\mathcal{G}}(x, y) \subset \mathcal{D}_{\mathcal{G}}(x, y)$.

The $\left(M, A_{1}, \ldots, A_{l}\right)$-graded ring $\mathbb{P}$ is the polynomial ring generated over $\mathbb{F}_{2}$ by the variables $U_{i, j}$ for $i \in\{1, \ldots, l\}, j \in\left\{1, \ldots, k_{i}\right\}$, and $C F_{\mathcal{G}}$ is the $\mathbb{F}_{2}\left[U_{1}, \ldots, U_{l}\right]-$ module freely generated over $\mathbb{P}$ by $\mathcal{S}_{\mathcal{G}}$, where the $U_{i}$-action is multiplication by $U_{i, s_{i}}$. The $U_{i, j}$-equivariant $(-1,0, \ldots, 0)$-graded boundary map, evaluated on a state $x$, is

$$
\partial x=\sum_{y \in \mathcal{S}_{\mathcal{G}}} y \sum_{\substack{R \in \mathcal{R}_{\mathcal{G}}(x, y) \\ n_{z}(R)=0}} \prod_{l, j} U_{l, j}^{n_{w_{l, j}}(R)} .
$$

\subsection{Changing the complex structure}

Let $J_{S}$ and $J_{S}^{\prime}$ be the constant paths of almost complex structures on $\operatorname{Sym}^{n}(T)$ induced from two complex structure on $T$. We know that $C F_{\left(\mathcal{G}, J_{s}\right)}$ and $C F_{\left(\mathcal{G}, J_{s}^{\prime}\right)}$ are the same object; therefore, it is not unnatural to expect the naturality map $F_{\left(\mathcal{G}, J_{S}\right),\left(\mathcal{G}, J_{s}^{\prime}\right)}$ in $\operatorname{Mor}_{K\left(\mathcal{A}_{l}\right)}\left(C F_{\left(\mathcal{G}, J_{s}\right)}, C F_{\left(\mathcal{G}, J_{s}^{\prime}\right)}\right)$ to be the identity map. This is indeed the case.

Theorem 5.2 If $J_{S}$ and $J_{s}^{\prime}$ are two constant paths of almost complex structures on $\operatorname{Sym}^{n}(T)$ induced from two complex structures on $T$, then $F_{\left(\mathcal{G}, J_{s}\right),\left(\mathcal{G}, J_{s}^{\prime}\right)}=\mathrm{Id}$.

Sketch of a proof This is a direct consequence of [13, Proof of Theorem 6.1], adapted to our present setting, where any Whitney disk $\varphi \in \pi_{2}(x, y)$ whose shadow $D(\varphi) \in \mathcal{D}_{\mathcal{G}}(x, y)$ is positive and which satisfies $\mu(\varphi) \leq 0$ is a trivial one.

\subsection{Commutation}

Commutation comes in two flavors, horizontal commutation and vertical commutation. The story for vertical commutation can be guessed from the story of horizontal commutation by reversing the roles of $\alpha$ and $\beta$; vertical commutations are also described 


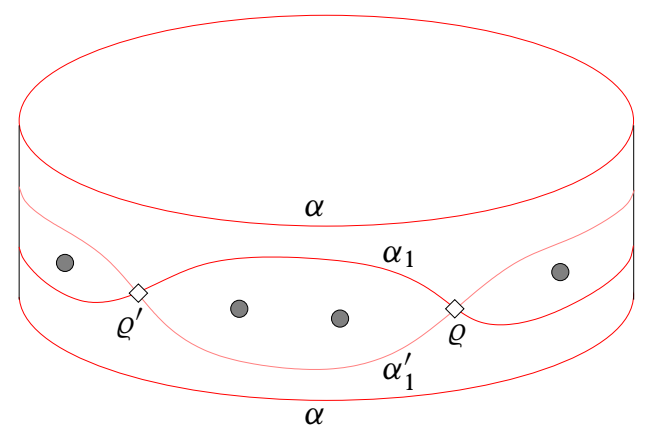

Figure 5.1: Horizontal commutation

in full detail in [8], from where much of the material for this subsection is derived; so for now, let us only talk about horizontal commutation.

A horizontal commutation is a pair of grid diagrams $\left(\mathcal{G}, \mathcal{G}^{\prime}\right)$ drawn on the same torus such that $\mathcal{G}^{\prime}$ can be obtained from $\mathcal{G}$ by changing exactly one $\alpha$-circle in the following manner: if the circle $\alpha_{1}$ in $\mathcal{G}$ is changed to the circle $\alpha_{1}^{\prime}$ in $\mathcal{G}^{\prime}$, then some neighborhood of $\alpha_{1} \cup \alpha_{1}^{\prime}$ must be homeomorphic to the region shown in Figure 5.1, where the four black dots are two $z$ markings and two $w$ markings. The two points in $\alpha_{1} \cap \alpha_{1}^{\prime}$ are marked as $\varrho$ and $\varrho^{\prime}$. The $\beta$-circles are not shown, but we assume that they avoid both $\varrho$ and $\varrho^{\prime}$.

Commutation induces the naturality map $F_{\mathcal{G}, \mathcal{G}^{\prime}} \in \operatorname{Mor}_{K\left(\mathcal{A}_{l}\right)}\left(C F_{\mathcal{G}}, C F_{\mathcal{G}^{\prime}}\right)$. Allow us to explain this map in grid diagram terminology.

For $x \in \mathcal{S}_{\mathcal{G}}$ and $y \in \mathcal{S}_{\mathcal{G}^{\prime}}$, a pentagon $P$ joining $x$ to $y$ is a 2-chain generated by the components of $T \backslash\left(\alpha \cup \alpha^{\prime} \cup \beta\right)$ such that each component appears with coefficient 0 or 1 , the closure of the union of the coefficient-1 components is an embedded pentagon one of whose vertices is $\varrho$, the embedded pentagon does not contain any coordinates of $x$ or $y$ in its interior, the northeast and southwest corners of the embedded pentagon are coordinates of $x$, and the northwest and southeast corners of the embedded pentagon are coordinates of $y$. The set of all pentagons joining $x$ to $y$ is denoted by $\varrho \mathcal{P}_{\mathcal{G}, \mathcal{G}^{\prime}}(x, y)$. We have a $U_{i, j}$-equivariant $(0,0, \ldots, 0)$-graded chain map $\varrho_{\mathcal{F}_{\mathcal{G}} \mathcal{G}^{\prime}}: C F_{\mathcal{G}} \rightarrow C F_{\mathcal{G}^{\prime}}$, which when evaluated on $x \in \mathcal{S}_{\mathcal{G}}$ is given by

$$
\varrho \widetilde{F}_{\mathcal{G}, \mathcal{G}^{\prime}}(x)=\sum_{y \in \mathcal{S}_{\mathcal{G}^{\prime}}} y \sum_{\substack{P \in_{\rho} \mathcal{P}_{\mathcal{G}, \mathcal{G}^{\prime}}(x, y) \\ n_{z}(P)=0}} \prod_{l, j} U_{l, j}^{n_{w_{l}, J}(P)} .
$$

This chain map depends on the location of the point $\varrho$, namely which component of $T \backslash \beta$ contains $\varrho$. Similarly, we get another chain map $\varrho^{\prime} \widetilde{F}_{\mathcal{G}^{\prime}, \mathcal{G}}: C F_{\mathcal{G}^{\prime}} \rightarrow C F_{\mathcal{G}}$, which depends on the location of the point $\varrho^{\prime}$. By [8, Proposition 3.2], any of the maps $\varrho^{\prime} \widetilde{F}_{\mathcal{G}^{\prime}, \mathcal{G}}$ 


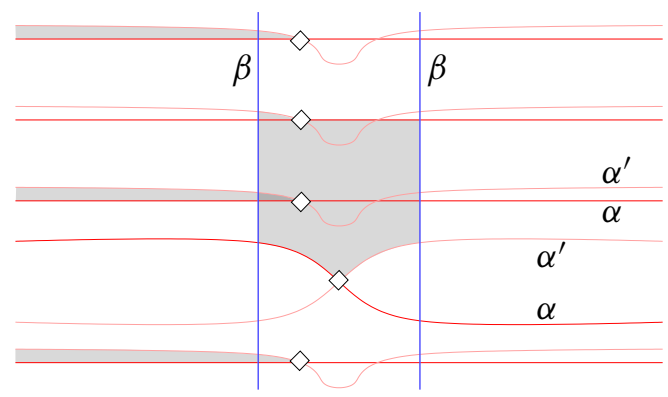

Figure 5.2: Horizontal commutation

is an inverse for any of the maps $\varrho \widetilde{F}_{\mathcal{G}, \mathcal{G}^{\prime}}$ in the homotopy category $K\left(\mathcal{A}_{l}\right)$. Therefore, we get a well-defined map $\widetilde{F}_{\mathcal{G}, \mathcal{G}^{\prime}} \in \operatorname{Mor}_{K\left(\mathcal{A}_{l}\right)}\left(C F_{\mathcal{G}}, C F_{\mathcal{G}^{\prime}}\right)$ which is independent of $\varrho$. It is not hard to check that this map $\widetilde{F}_{\mathcal{G}, \mathcal{G}^{\prime}}$, defined in terms of pentagons in the torus $T$, is same as the map $F_{\left(\mathcal{G}, J_{S}\right),\left(\mathcal{G}^{\prime}, J_{S}\right)}$, defined using holomorphic triangles in $\operatorname{Sym}^{n}(T)$. We give a direct verification below.

Given a commutation diagram, we can perturb $\alpha^{\prime}$ to ensure that each $\alpha^{\prime}$-circle intersects its corresponding $\alpha$-circle in exactly two points; furthermore, except for $\varrho^{\prime}$, we can ensure that each of the intersection points lies in the component of $T \backslash \beta$ that contains the point $\varrho$. The situation is illustrated in Figure 5.2. Let $\Theta$ be the top-dimensional generator in the Heegaard diagram $\left(T, \alpha^{\prime}, \alpha\right)$; the coordinates of $\Theta$ are shown (note, $\varrho$ is one of the coordinates).

For $x \in \mathcal{S}_{\mathcal{G}}$ and $y \in \mathcal{S}_{\mathcal{G}^{\prime}}$, if $D$ is a 2-chain generated by the components of $T \backslash\left(\alpha \cup \alpha^{\prime} \cup \beta\right)$ such that $\partial\left(\left.\partial D\right|_{\alpha}\right)=\Theta-x$ and $\partial\left(\left.\partial D\right|_{\alpha^{\prime}}\right)=y-\Theta$, then $D$ is called a domain joining $x, \Theta$ and $y$. If $\varphi \in \pi_{2}(x, \Theta, y)$ is a Whitney triangle connecting $x, \Theta$ and $y$ in $\operatorname{Sym}^{n}(T)$, then its shadow $D(\varphi)$ is a domain joining $x, \Theta$ and $y$. Conversely, given any domain $D$ joining $x, \Theta$ and $y$, there is a unique Whitney triangle $\varphi \in \pi_{2}(x, \Theta, y)$ such that $D=D(\varphi)$.

It is easy to see that each pentagon in $\varrho^{\mathcal{P}_{\mathcal{G}, \mathcal{G}^{\prime}}}(x, y)$ gives rise to exactly two positive domains joining $x, \Theta$ and $y$. Let us call such domains twin pentagonal domains. One such pentagonal domain is shown in Figure 5.2.

Lemma 5.3 If $\varphi \in \pi_{2}(x, \Theta, y)$ is a Whitney triangle with $\mu(\varphi)=0$ and $D(\varphi)$ positive, then $D(\varphi)$ is a pentagonal domain. Conversely, if $\varphi, \varphi^{\prime} \in \pi_{2}(x, \Theta, y)$ are Whitney triangles such that $D(\varphi)$ and $D\left(\varphi^{\prime}\right)$ are twin pentagonal domains, then $\mu(\varphi)=\mu\left(\varphi^{\prime}\right)=0$ and $|\mathcal{M}(\varphi)|+\left|\mathcal{M}\left(\varphi^{\prime}\right)\right|=1$. Therefore, the triangle map $F_{\mathcal{G}, \mathcal{G}^{\prime}}$ agrees with $\widetilde{F}_{\mathcal{G}, \mathcal{G}^{\prime}}$. 
Proof For the first direction, assume $\varphi \in \pi_{2}(x, \Theta, y)$ is a Whitney triangle with $\mu(\varphi)=0$ and $D(\varphi)$ positive. Let $B$ be the union of the $2 n$ bigonal components of $T \backslash\left(\alpha \cup \alpha^{\prime}\right)$. Let $D^{\prime}$ be the unique domain joining some state $y^{\prime} \in \mathcal{S}_{\mathcal{G}}$ and $\Theta$ and $y$ such that $D^{\prime}$ is supported in $B$ and $\left.\partial D^{\prime}\right|_{\alpha^{\prime}}=\left.\partial D(\varphi)\right|_{\alpha^{\prime}}$. Using the Maslov index formula from [18], it is easy to check that the Maslov index of $D^{\prime}$ is -1 . Therefore, $\widetilde{D}=D(\varphi)-D^{\prime}$ is a Maslov index-1 domain in $\mathcal{D}_{\mathcal{G}}\left(x, y^{\prime}\right)$. Furthermore, since $D^{\prime}$ has coefficient zero outside $B$ and $D(\varphi)$ is positive, $\widetilde{D}$ is a positive domain as well. Theorem 5.1 tells us that $\widetilde{D}$ is a rectangle in $\mathcal{G}$. However, we know that $D(\varphi)=\widetilde{D}+D^{\prime}$ is also a positive domain. Therefore, the rectangle $\widetilde{D}$ can only be in certain configurations. By analyzing them carefully, we see that those are precisely the configurations for which $D(\varphi)$ is a pentagonal domain.

The other direction is fairly straightforward. Let $\varphi, \varphi^{\prime} \in \pi_{2}(x, \Theta, y)$ be Whitney triangles such that $D(\varphi)$ and $D\left(\varphi^{\prime}\right)$ are twin pentagonal domains. A direct computation reveals that the Maslov index of any pentagonal domain is zero. To show that $|\mathcal{M}(\varphi)|+\left|\mathcal{M}\left(\varphi^{\prime}\right)\right|=1$, following standard practice, we will use Lipshitz's cylindrical reformulation [4]; in the cylindrical version, we will count the number of holomorphic embeddings of surfaces $F \hookrightarrow T \times\left\{z \in \mathbb{C}|| z \mid \leq 1, z^{3} \neq 1\right\}$ satisfying certain conditions, such as that the image of the projection onto the first factor is either $D(\varphi)$ or $D\left(\varphi^{\prime}\right)$, and the projection onto the second factor is an $n$-sheeted cover of $\left\{z \in \mathbb{C}|| z \mid \leq 1, z^{3} \neq 1\right\}$ with exactly one branch point (the number of branch points can be figured out from another formula in [18]). Therefore, $F$ must be a disjoint union of $n-2$ copies of a disk punctured at three boundary points (call them 3-gons) and a single copy of a disk punctured at six boundary points (call it a 6-gon). Therefore, the moduli space $\mathcal{M}(\varphi) \sqcup \mathcal{M}\left(\varphi^{\prime}\right)$ is the product of the $n-1$ moduli spaces coming from these $n-1$ components. It is a fairly easy exercise in complex analysis to check that the moduli space of embeddings of a 3 -gon contains exactly one point.

To show the moduli space of embeddings of the 6 -gon into $T \times\left\{z \in \mathbb{C}|| z \mid \leq 1, z^{3} \neq 1\right\}$ contains an odd number of points, let us do a model calculation. If $\varrho$ lies in the southern portion of the pentagonal domains $D(\varphi)$ and $D\left(\varphi^{\prime}\right)$, then one of $D(\varphi)$ and $D\left(\varphi^{\prime}\right)$ looks like the shaded hexagonal region in Figure 5.2, while the other one is its twin; if $\varrho$ lies in the northern portion of the pentagonal domain, then $D(\varphi)$ and $D\left(\varphi^{\prime}\right)$ have similar but different shapes. Call $D(\varphi)$ and $D\left(\varphi^{\prime}\right)$ the original twin pentagonal domains. Now consider the index-2 grid diagram $\mathcal{G}_{0}$ for the 2-component unlink. There are two states and they lie in different Maslov gradings. Do a horizontal commutation to obtain the grid diagram $\mathcal{G}_{1}$, which also has two states, lying in different Maslov gradings. The triangle map must be an isomorphism, and we have already seen that the shadow of each Whitney triangle must be a pentagonal domain. There are exactly two sets of twin pentagonal domains in this model commutation diagram. Consider 
the twin pentagonal domains that have the same shape as the original twin pentagonal domains. Call them the model twin pentagonal domains. Choose a complex structure on the model twin pentagonal domains which matches the one on the original twin pentagonal domains. Since the triangle map for the model commutation is a graded isomorphism, the moduli space corresponding to the model twin pentagonal domains, and hence the moduli space corresponding to the original twin pentagonal domains, must contain an odd number of points.

\subsection{Stabilization and destabilization}

Stabilization for grid diagrams is a move that converts a grid diagram of index $n$ to a grid diagram of index $n+1$. There are several variants (four or eight, depending on how we count them) of stabilization. However, we are mostly concerned with only one of these configurations, so let us describe it in detail.

Let $\mathcal{G}=(T, \alpha, \beta, z, w)$ be an index- $n$ grid diagram where the link component $L_{1}$ contains the $2 k_{1}$ markings $z_{1,1}, \ldots, z_{1, k_{1}}, w_{1,1}, \ldots, w_{1, k_{1}}$. Let $\mathcal{G}^{\prime}=\left(T, \alpha^{\prime}, \beta^{\prime}, z^{\prime}, w^{\prime}\right)$ be an index- $(n+1)$ grid diagram representing $L$ and satisfying the following: $L_{1}$ contains two additional markings $z_{1, k_{1}+1}$ and $w_{1, k_{1}+1}$; there is an $\alpha^{\prime}$-circle $\alpha_{n+1}$ and a $\beta^{\prime}$ circle $\beta_{n+1}$, intersecting each other a point $\varrho$ with $z_{1, k_{1}+1}$ lying immediately to the southeast of $\varrho$ and $w_{1, k_{1}+1}$ lying immediately to the northeast of $\varrho$; and $\mathcal{G}$ can be obtained from $\mathcal{G}^{\prime}$ by deleting $z_{1, k_{1}+1}, w_{1, k_{1}+1}, \alpha_{n+1}$ and $\beta_{n+1}$. Getting $\mathcal{G}^{\prime}$ from $\mathcal{G}$ is an instance of stabilization.

Since $\alpha \subset \alpha^{\prime}$ and $\beta \subset \beta^{\prime}, \mathcal{S}_{\mathcal{G}}$ can be identified with the subset of $\mathcal{S}_{\mathcal{G}^{\prime}}$ consisting of the states that contain the point $\varrho$. Furthermore, since $C F_{\mathcal{G}}$ is freely generated by $\mathcal{S}_{\mathcal{G}}$ over the $\mathbb{F}_{2}$-algebra $\mathbb{P}$ and $C F_{\mathcal{G}^{\prime}}$ is freely generated by $\mathcal{S}_{\mathcal{G}^{\prime}}$ over the $\mathbb{F}_{2}$-algebra $\mathbb{P}^{\prime}=\mathbb{P} \otimes \mathbb{F}_{2}\left[U_{1, k_{1}+1}\right]$, there is a natural inclusion map $\iota$ : $C F_{\mathcal{G}} \hookrightarrow C F_{\mathcal{G}^{\prime}}$ (note, $\iota$ is just a $\mathbb{P}$-module map, it in general is not a chain map). With this in mind, let us describe the naturality map $F_{\mathcal{G}, \mathcal{G}^{\prime}}$.

A northeast snail domain centered at $\varrho$ is a positive domain $D \in \mathcal{D}_{\mathcal{G}^{\prime}}(x, y)$ for $x, y \in \mathcal{S}_{\mathcal{G}^{\prime}}$ such that the following hold: $\partial D$ is an immersed circle in $T$; each coordinate of $x$ and $y$, except possibly $\varrho$, appears with coefficient 0 or $\frac{1}{4}$ in $D$; and there is some $m \geq 0$ such that the coefficient of $D$ is $m+1$ immediately to the northeast of $\varrho$ and is $m$ in the other three squares adjacent to $\varrho$. Let $\mathcal{L}_{\varrho}^{1}(x, y)$ denote the set of all northeast snail domain centered at $\varrho$ joining $x$ to $y$. We have shown some elements of $\mathcal{L}_{\varrho}^{1}(x, y)$ in the first row of Figure 5.3. 


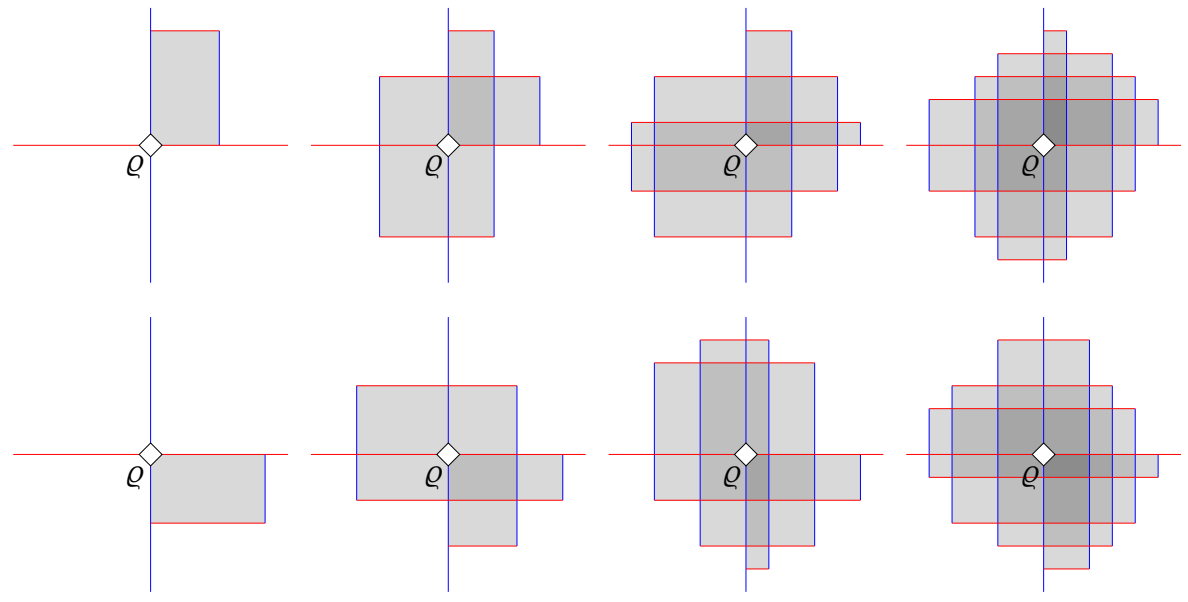

Figure 5.3: Snail domains

Let us define a $\mathbb{P}$-module $(0,0, \ldots, 0)$-graded chain map $\widetilde{F}_{\mathcal{G}, \mathcal{G}^{\prime}}: C F_{\mathcal{G}} \rightarrow C F_{\mathcal{G}^{\prime}}$ as follows. For $x \in \mathcal{S}_{\mathcal{G}}$, if $\iota(x)$ denotes the corresponding state in $\mathcal{S}_{\mathcal{G}^{\prime}}$, then

$$
\tilde{F}_{\mathcal{G}, \mathcal{G}^{\prime}}(x)=\sum_{y \in \mathcal{S}_{\mathcal{G}^{\prime}}} y \sum_{\substack{D \in \mathcal{L}_{\varrho}^{1}(\iota(x), y) \\ n_{z}(D)=n_{z_{1, k}+1}(D)}} \prod_{\substack{l, J \\(l, J) \neq\left(1, k_{1}+1\right)}} U_{l, J}^{n_{w_{l, j}}(D)} .
$$

There are similar maps for the other types of stabilization, which can be defined in terms of similar-looking snail domains. All these snail domains are very similar to the snail domains that appear in [8]; following their analysis, it is not very hard to see that each of the stabilization maps, and in particular the above one, is a chain map. Before we show that this map is the same as the naturality map $F_{\mathcal{G}, \mathcal{G}^{\prime}}$ in $\operatorname{Mor}_{K\left(\mathcal{A}_{l}\right)}\left(C F_{\mathcal{G}}, C F_{\mathcal{G}^{\prime}}\right)$, let us talk a little bit about destabilization.

Destabilization for grid diagrams is the reverse move of stabilization. Once again, there are several variants and, once again, we will concentrate on only one. The starting grid diagram $\mathcal{G}=(T, \alpha, \beta, z, w)$ has index $n+1$ and the link component $L_{1}$ contains the $2 k_{1}+2$ markings $z_{1,1}, \ldots, z_{1, k_{1}+1}, w_{1,1}, \ldots, w_{1, k_{1}+1}$. Furthermore, assume that there is an $\alpha$-circle $\alpha_{n+1}$ and a $\beta$-circle $\beta_{n+1}$, intersecting each other a point $\varrho$, such that $z_{1, k_{1}+1}$ lies immediately to the southeast of $\varrho$ and $w_{1, k_{1}+1}$ lies immediately to the southwest of $\varrho$; let $w_{1, d}$ be the $w$-marking that lies in the same component of $T \backslash \beta$ as $z_{1, k_{1}+1}$. The index- $n$ grid diagram $\mathcal{G}^{\prime}$ is obtained from $\mathcal{G}$ by deleting $z_{1, k_{1}+1}, w_{1, k_{1}+1}, \alpha_{n+1}$ and $\beta_{n+1}$; this is an instance of destabilization.

Once again, there is a natural inclusion $\iota: \mathcal{S}_{\mathcal{G}^{\prime}} \hookrightarrow \mathcal{S}_{\mathcal{G}}$, which induces a $\mathbb{P}^{\prime}$-module map $\iota: C F_{\mathcal{G}^{\prime}} \hookrightarrow C F_{\mathcal{G}}$. For $x, y \in \mathcal{S}_{\mathcal{G}}$, a southeast snail domain centered at $\varrho$ is a positive 
domain $D \in \mathcal{D}_{\mathcal{G}}(x, y)$ such that $\partial D$ is an immersed circle in $T$, each coordinate of $x$ and $y$, except possibly $\varrho$, appears with coefficient 0 or $\frac{1}{4}$ in $D$, and there is some $m \geq 0$ such that the coefficient of $D$ is $m+1$ immediately to the southeast of $\varrho$ and is $m$ in the other three squares adjacent to $\varrho$. Let $\mathcal{L}_{\varrho}^{2}(x, y)$ denote the set of all southeast snail domain centered at $\varrho$ joining $x$ to $y$. Some elements of $\mathcal{L}_{\varrho}^{2}(x, y)$ are shown in the second row of Figure 5.3. In [8], a $\mathbb{P}^{\prime}$-module $(0,0, \ldots, 0)$-graded chain map $\widetilde{F}_{\mathcal{G}, \mathcal{G}^{\prime}}: C F_{\mathcal{G}} \rightarrow C F_{\mathcal{G}^{\prime}}$ is defined as follows:

$$
\widetilde{F}_{\mathcal{G}, \mathcal{G}^{\prime}}\left(U_{1, k_{1}+1}^{m} x\right)=\sum_{y \in \mathcal{S}_{\mathcal{G}^{\prime}}} y \sum_{\substack{D \in \mathcal{L}_{\varrho}^{2}(x, l(y)) \\ n_{z}(D)=n_{z_{1}, k_{1}+1}(D)}} U_{1, d}^{m} \prod_{l, j} U_{l, j}^{n_{w_{l, j}}(D)} .
$$

There are similar chain maps for the other types of destabilization. Manolescu and Ozsváth [6] show that, for each configuration, the destabilization map $\widetilde{F}_{\mathcal{G}, \mathcal{G}^{\prime}}$, defined in terms of the snail domains, is the same as the naturality map $F_{\mathcal{G}, \mathcal{G}^{\prime}}$ in $\operatorname{Mor}_{K\left(\mathcal{A}_{l}\right)}\left(C F_{\mathcal{G}}, C F_{\mathcal{G}^{\prime}}\right)$. This implies that the same is true for the stabilization maps.

Lemma 5.4 If $\mathcal{G}^{\prime}$ is obtained from $\mathcal{G}$ by a stabilization, then the stabilization map $\widetilde{F}_{\mathcal{G}, \mathcal{G}^{\prime}}$, defined in terms of the snail domains, is the same as the naturality map $F_{\mathcal{G}, \mathcal{G}^{\prime}}$ in $\operatorname{Mor}_{D\left(\mathcal{A}_{l}\right)}\left(C F_{\mathcal{G}}, C F_{\mathcal{G}^{\prime}}\right)$.

Proof By naturality, we know that $F_{\mathcal{G}, \mathcal{G}^{\prime}} F_{\mathcal{G}^{\prime}, \mathcal{G}}=\mathrm{Id}$ in $K\left(\mathcal{A}_{l}\right)$. From [6] we know that $F_{\mathcal{G}^{\prime}, \mathcal{G}}=\widetilde{F}_{\mathcal{G}^{\prime}, \mathcal{G}}$ in $K\left(\mathcal{A}_{l}\right)$. Finally, it is easy to check that the chain map $\widetilde{F}_{\mathcal{G}^{\prime}, \mathcal{G}} \widetilde{F}_{\mathcal{G}, \mathcal{G}^{\prime}}$ actually equals the identity map on $C F_{\mathcal{G}}$. Therefore,

$$
\tilde{F}_{\mathcal{G}, \mathcal{G}^{\prime}}=F_{\mathcal{G}, \mathcal{G}^{\prime}} F_{\mathcal{G}^{\prime}, \mathcal{G}} \widetilde{F}_{\mathcal{G}, \mathcal{G}^{\prime}}=F_{\mathcal{G}, \mathcal{G}^{\prime}} \widetilde{F}_{\mathcal{G}^{\prime}, \mathcal{G}} \widetilde{F}_{\mathcal{G}, \mathcal{G}^{\prime}}=F_{\mathcal{G}, \mathcal{G}^{\prime}}
$$

\subsection{Renumbering}

There is yet a third type of grid move, namely renumbering the $w$ - and $z$-markings. Since we are only concerned with the link component $L_{1}$, let us only consider the renumbering of the $w_{1}$ - and the $z_{1}$-markings. Let us start with a grid diagram $\mathcal{G}$ with $k_{1} \quad w_{1}$-markings and $k_{1} z_{1}$-markings. Fix $\sigma, \tau \in \mathfrak{S}_{k_{1}}$. Let $\mathcal{G}^{\prime}$ be the grid diagram obtained from $\mathcal{G}$ by renaming $w_{1, i}$ as $w_{1, \sigma(i)}$ and $z_{1, i}$ as $z_{1, \tau(i)}$ for $1 \leq i \leq k_{1}$; furthermore, if $w_{1, s_{1}}$ is the special $w_{1}$-marking in $\mathcal{G}$, then $w_{1, \sigma\left(s_{1}\right)}$ is the special $w_{1}^{\prime}$-marking in $\mathcal{G}^{\prime}$.

The grid diagrams $\mathcal{G}$ and $\mathcal{G}^{\prime}$ represent the same pointed link. The naturality map $F_{\mathcal{G}, \mathcal{G}^{\prime}}$ is $U_{i, j}$-equivariant for $i \neq 1$ and sends $x \prod_{j} U_{1, j}^{m_{j}}$ to $x \prod_{j} U_{1, \sigma(j)}^{m_{j}}$. This is simply because the $U_{i, j}$ variables are not indexed by pairs of integers $i, j$, but are rather indexed by the $w$-markings themselves. Indeed, we should have written $U_{i, j}$ as $U_{w_{i, j}}$. Precise notations lead to triple subscripts (as in $U_{w_{i, s_{i}}}$ !), so we have chosen to avoid them. 


\subsection{Changing the special marking}

Let $\mathcal{G}=\mathcal{G}_{1}$ be a grid diagram for the pointed link $(L, p)$ such that $s_{1}=1$ (ie the basepoint $p_{1} \in L_{1}$ is represented by the $w$-marking $\left.w_{1,1}\right)$ and the markings that appear in $L_{1}$ are, in order, $w_{1,1}, z_{1,1}, \ldots, w_{1, k_{1}}, z_{1, k_{1}}$ with $k_{1}>1$. For $2 \leq j \leq k_{1}$, let $\mathcal{G}_{j}$ be the grid diagram where $s_{1}=j$ but which is otherwise identical to $\mathcal{G}$. Observe that the grid diagrams $\mathcal{G}_{j}$ represent the same link, but not the same pointed link. The chain complexes $C F_{\mathcal{G}_{j}}$ are identical as $\mathbb{F}_{2}\left[U_{2}, \ldots, U_{l}\right]$-modules; the $U_{1}$-action is multiplication by $U_{1, j}$.

For distinct $j, j^{\prime} \in\left\{1, \ldots, k_{1}\right\}$, we will define $U_{i}$-equivariant chain maps $f_{j, j^{\prime}}$ from $C F_{\mathcal{G}_{j}}$ to $C F_{\mathcal{G}_{j^{\prime}}}$. Define $c_{j, j^{\prime}}: C F_{\mathcal{G}_{j}} \rightarrow C F_{\mathcal{G}_{j^{\prime}}}$ as follows: it is $U_{l, j}$-equivariant for $(l, j) \neq(1, j)$ and sends $U_{1, j}^{m} x$ to $U_{1, j^{\prime}}^{m} x$. It is clearly an $\mathbb{F}_{2}\left[U_{1}, \ldots, U_{l}\right]$-module map, but in general is not a chain map. Let $J\left(j, j^{\prime}\right) \subseteq\left\{1, \ldots, k_{1}\right\}$ be defined by: $J \in J\left(j, j^{\prime}\right)$ if and only if $z_{1, j}$ appears in the arc joining $w_{1, j}$ to $w_{1, j^{\prime}}$ in the oriented link component $L_{1}$. Define

$$
f_{j, j^{\prime}}=c_{j, j^{\prime}} \Phi_{1, j} \sum_{j \in J\left(j, j^{\prime}\right)} \Psi_{1, j} .
$$

Theorem 5.5 The map $f_{j, j^{\prime}}$, as defined above, is an $\mathbb{F}_{2}\left[U_{1}, \ldots, U_{l}\right]$-module chain map from $C F_{\mathcal{G}_{j}}$ to $C F_{\mathcal{G}_{j^{\prime}}}$. Furthermore, $f_{k_{1}, 1} f_{1, k_{1}}=\mathrm{Id}+\Phi_{1} \Psi_{1}=\mathrm{Id}+\Psi_{1} \Phi_{1}$ and, for $2 \leq j \leq k_{1}-1, f_{j, j+1} f_{1, j}=f_{1, j+1}$ in $K\left(\mathcal{A}_{l}\right)$

Proof The maps $\Phi_{i, j}$ and $\Psi_{i, j}$ are $U_{l, j}$-equivariant. The map $c_{j, j^{\prime}}$ is also $U_{i-}$ equivariant since $\left[c_{j, j^{\prime}}: U_{1}\right]=c_{j, j^{\prime}} U_{1}+U_{1} c_{j, j^{\prime}}=c_{j, j^{\prime}} U_{1, j}+U_{1, j^{\prime}} c_{j, j^{\prime}}=0$. Therefore, the maps $f_{j, j^{\prime}}$ are $U_{i}$-equivariant.

To see that $f_{j, j^{\prime}}$ is a chain map, recall the commutator relations: $\left[\Phi_{1, j}: \partial\right]=0$ and $\left[\Psi_{1, j}: \partial\right]=U_{1, j}+U_{1, j+1}$ (the second index being numbered modulo $k_{1}$ ). It is also easy to see that $\left[c_{j, j^{\prime}}: \partial\right]=\left(U_{1, j}+U_{1, j^{\prime}}\right) c_{j, j^{\prime}} \Phi_{1, j}$ (since $w_{1, j}$ can appear in a rectangle at most once). Therefore,

$$
\begin{aligned}
{\left[f_{j, j^{\prime}}: \partial\right] } & =\left(\left[c_{j, j^{\prime}}: \partial\right] \Phi_{1, j} \sum_{j \in J\left(j, j^{\prime}\right)} \Psi_{1, j}\right)+\left(c_{j, j^{\prime}}\left[\Phi_{1, j}: \partial\right] \sum_{J \in J\left(j, j^{\prime}\right)} \Psi_{1, j}\right) \\
& +\left(c_{j, j^{\prime}} \Phi_{1, j} \sum_{j \in J\left(j, j^{\prime}\right)}\left[\Psi_{1, j}: \partial\right]\right) \\
& =\left(\left(U_{1, j}+U_{1, j^{\prime}}\right) c_{j, j^{\prime}} \Phi_{1, j}^{2} \sum_{j \in J\left(j, j^{\prime}\right)} \Psi_{1, j}\right)+\left(c_{j, j^{\prime}} \Phi_{1, j}\left(U_{1, j}+U_{1, j^{\prime}}\right)\right) \\
& =0+c_{j, j^{\prime}}\left(U_{1, j}+U_{1, j^{\prime}}\right) \Phi_{1, j} \quad \text { (since } \Phi_{1, j}^{2}=0 \text { in grid diagrams) } \\
& =0 .
\end{aligned}
$$


For the second part of the theorem, let $\operatorname{Id}_{j, j^{\prime}}$ be the identity map from $C F_{\mathcal{G}_{j}}$ to $C F_{\mathcal{G}_{j^{\prime}}}$. It is not $U_{1}$-equivariant; indeed, $\left[\operatorname{Id}_{j, j^{\prime}}: U_{1}\right]=\left(U_{1, j}+U_{1, j^{\prime}}\right) \operatorname{Id}_{j, j^{\prime}}$. Define

$$
K_{j, j^{\prime}}: C F_{\mathcal{G}_{j}} \rightarrow C F_{\mathcal{G}_{j^{\prime}}}
$$

to be $U_{l, j}$-equivariant for $(l, j) \neq(1, j)$ and send $U_{1, j}^{m} x$ to $\left(\left(U_{j}^{m}+U_{j^{\prime}}^{m}\right) /\left(U_{j}+U_{j^{\prime}}\right)\right) x$. It is easy to see that $\left[K_{j, j^{\prime}}: U_{1}\right]=U_{1, j^{\prime}} K_{j, j^{\prime}}+K_{j, j^{\prime}} U_{1, j}=\operatorname{Id}_{j, j^{\prime}}$; furthermore, $\left[K_{j, j^{\prime}}: \partial\right]=c_{j, j^{\prime}} \Phi_{1, j}$.

We have

$$
\begin{aligned}
& f_{k_{1}, 1} f_{1, k_{1}}+\Phi_{1} \Psi_{1} \\
& \quad=c_{k_{1}, 1} \Phi_{1, k_{1}} \Psi_{1, k_{1}} c_{1, k_{1}} \Phi_{1,1}\left(\Psi_{1,1}+\cdots+\Psi_{1, k_{1}-1}\right)+\Phi_{1,1}\left(\Psi_{1,1}+\cdots+\Psi_{1, k_{1}}\right) \\
& \quad=\left(c_{k_{1}, 1} \Phi_{1, k_{1}} \Psi_{1, k_{1}} \operatorname{Id}_{1, k_{1}}+\mathrm{Id}\right) c_{1,1} \Phi_{1,1}\left(\Psi_{1,1}+\cdots+\Psi_{1, k_{1}-1}\right)+c_{1,1} \Phi_{1,1} \Psi_{1, k_{1}}
\end{aligned}
$$

and, for $2 \leq j \leq k_{1}-1$,

$$
\begin{aligned}
& f_{1, j} f_{j, j+1}+f_{1, j+1} \\
& =c_{j, j+1} \Phi_{1, j} \Psi_{1, j} c_{1, j} \Phi_{1,1}\left(\Psi_{1,1}+\cdots+\Psi_{1, j-1}\right)+c_{1, j+1} \Phi_{1,1}\left(\Psi_{1,1}+\cdots+\Psi_{1, j}\right) \\
& =\left(c_{j, j+1} \Phi_{1, j} \Psi_{1, j} \operatorname{Id}_{j+1, j}+\mathrm{Id}\right) c_{1, j+1} \Phi_{1,1}\left(\Psi_{1,1}+\cdots+\Psi_{1, j-1}\right) \\
& \quad+c_{1, j+1} \Phi_{1,1} \Psi_{1, j} .
\end{aligned}
$$

Therefore, for $2 \leq j \leq k_{1}$, we are interested in the map

$$
\begin{aligned}
g_{j}=\left(c_{j, j+1} \Phi_{1, j} \Psi_{1, j} \operatorname{Id}_{j+1, j}+\operatorname{Id}\right) c_{1, j+1} \Phi_{1,1}\left(\Psi_{1,1}+\cdots+\Psi_{1, j-1}\right) & +c_{1, j+1} \Phi_{1,1} \Psi_{1, j}
\end{aligned}
$$

We want to show that $g_{k_{1}}=\mathrm{Id}$ and $g_{j}=0$ for $j \leq k_{1}-1$ in $K\left(\mathcal{A}_{l}\right)$.

Consider the map $H_{j}=K_{j, j+1} \Psi_{1, j} \operatorname{Id}_{j+1, j} c_{1, j+1} \Phi_{1,1}\left(\Psi_{1,1}+\cdots+\Psi_{1, j-1}\right)$. We have

$$
\begin{aligned}
& \text { [ } \left.H_{j}: U_{1}\right] \\
& =\left[K_{j, j+1}: U_{1}\right] \Psi_{1, j} \operatorname{Id}_{j+1, j} c_{1, j+1} \Phi_{1,1}\left(\Psi_{1,1}+\cdots+\Psi_{1, j-1}\right) \\
& +K_{j, j+1} \Psi_{1, j}\left[\operatorname{Id}_{j+1, j}: U_{1}\right] c_{1, j+1} \Phi_{1,1}\left(\Psi_{1,1}+\cdots+\Psi_{1, j-1}\right) \\
& =\operatorname{Id}_{j, j+1} \Psi_{1, j} \operatorname{Id}_{j+1, j} c_{1, j+1} \Phi_{1,1}\left(\Psi_{1,1}+\cdots+\Psi_{1, j-1}\right) \\
& +K_{j, j+1} \Psi_{1, j}\left(U_{1, j}+U_{1, j+1}\right) \operatorname{Id}_{j+1, j} c_{1, j+1} \Phi_{1,1}\left(\Psi_{1,1}+\cdots+\Psi_{1, j-1}\right) \\
& =\operatorname{Id}_{j, j+1} \Psi_{1, j} \operatorname{Id}_{j+1, j} c_{1, j+1} \Phi_{1,1}\left(\Psi_{1,1}+\cdots+\Psi_{1, j-1}\right) \\
& +\operatorname{Id}_{j, j+1} \Psi_{1, j} \operatorname{Id}_{j+1, j} c_{1, j+1} \Phi_{1,1}\left(\Psi_{1,1}+\cdots+\Psi_{1, j-1}\right) \\
& =0 \text {. }
\end{aligned}
$$


Therefore, $H_{j}$ is a $U_{i}$-equivariant map from $C F_{\mathcal{G}}$ to $C F_{\mathcal{G}_{j+1}}$. We also have $\left[H_{j}: \partial\right]$

$$
\begin{aligned}
& =\left[K_{j, j+1}: \partial\right] \Psi_{1, j} \operatorname{Id}_{j+1, j} c_{1, j+1} \Phi_{1,1}\left(\Psi_{1,1}+\cdots+\Psi_{1, j-1}\right) \\
& +K_{j, j+1}\left[\Psi_{1, j}: \partial\right] \operatorname{Id}_{j+1, j} c_{1, j+1} \Phi_{1,1}\left(\Psi_{1,1}+\cdots+\Psi_{1, j-1}\right) \\
& \quad+K_{j, j+1} \Psi_{1, j} \operatorname{Id}_{j+1, j}\left[c_{1, j+1}: \partial\right] \Phi_{1,1}\left(\Psi_{1,1}+\cdots+\Psi_{1, j-1}\right) \\
& \quad+K_{j, j+1} \Psi_{1, j} \operatorname{Id}_{j+1, j} c_{1, j+1} \Phi_{1,1}\left(\left[\Psi_{1,1}: \partial\right]+\cdots+\left[\Psi_{1, j-1}: \partial\right]\right) \\
& =c_{j, j+1} \Phi_{1, j} \Psi_{1, j} \operatorname{Id}_{j+1, j} c_{1, j+1} \Phi_{1,1}\left(\Psi_{1,1}+\cdots+\Psi_{1, j-1}\right) \\
& +K_{j, j+1}\left(U_{1, j}+U_{1, j+1}\right) \operatorname{Id}_{j+1, j} c_{1, j+1} \Phi_{1,1}\left(\Psi_{1,1}+\cdots+\Psi_{1, j-1}\right) \\
& \quad+K_{j, j+1} \Psi_{1, j} \operatorname{Id}_{j+1, j}\left(U_{1,1}+U_{1, j+1}\right) c_{1, j+1} \Phi_{1,1}^{2}\left(\Psi_{1,1}+\cdots+\Psi_{1, j-1}\right) \\
& \quad+K_{j, j+1} \Psi_{1, j} \operatorname{Id}_{j+1, j} c_{1, j+1} \Phi_{1,1}\left(U_{1,1}+U_{1, j}\right) \\
& =c_{j, j+1} \Phi_{1, j} \Psi_{1, j} \operatorname{Id}_{j+1, j} c_{1, j+1} \Phi_{1,1}\left(\Psi_{1,1}+\cdots+\Psi_{1, j-1}\right) \\
& +\operatorname{Id}_{j, j+1} \operatorname{Id}_{j+1, j} c_{1, j+1} \Phi_{1,1}\left(\Psi_{1,1}+\cdots+\Psi_{1, j-1}\right) \\
& \quad+0+K_{j, j+1}\left(U_{1, j+1}+U_{1, j}\right) \Psi_{1, j} \operatorname{Id}_{j+1, j} c_{1, j+1} \Phi_{1,1} \\
& =\left(c_{j, j+1} \Phi_{1, j} \Psi_{1, j} \operatorname{Id}_{j+1, j}+\operatorname{Id}\right) c_{1, j+1} \Phi_{1,1}\left(\Psi_{1,1}+\cdots+\Psi_{1, j-1}\right) \\
& +\Psi_{1, j} c_{1, j+1} \Phi_{1,1} .
\end{aligned}
$$

Thus, $\left[H_{j}: \partial\right]+g_{j}=\Psi_{1, j} c_{1, j+1} \Phi_{1,1}+c_{1, j+1} \Phi_{1,1} \Psi_{1, j}$. We want to show that it id for $j=k_{1}$ and is zero for $j \leq k_{1}-1$ in $K\left(\mathcal{A}_{l}\right)$.

For $j \leq k_{1}-1$, consider the map $K_{1, j+1} \Psi_{1, j}+\Psi_{1, j} K_{1, j+1}$. It is straightforward to check that it is $U_{i}$-equivariant and its commutator with $\partial$ is

$$
\Psi_{1, j} c_{1, j+1} \Phi_{1,1}+c_{1, j+1} \Phi_{1,1} \Psi_{1, j} .
$$

For the last remaining case, consider the $U_{i}$-equivariant map $H: C F_{\mathcal{G}} \rightarrow C F_{\mathcal{G}}$ defined as follows:

$$
H(x)=\sum_{y \in \mathcal{S}_{\mathcal{G}}} y \sum_{\substack{R \in \mathcal{R}_{\mathcal{G}}(x, y) \\ n_{w_{1,1}}(R)=1 \\ n_{z}(R)=n_{z_{1, k_{1}}}(R)=1}} \prod_{(l, J) \neq(1,1)} U_{l, J}^{n_{w_{l, J}}(R)}
$$

It is easy to see that, since $k_{1}>1,[H: \partial]=\Phi_{1,1} \Psi_{1, k_{1}}+\Psi_{1, k_{1}} \Phi_{1,1}+$ Id. This concludes the proof.

\subsection{The main theorem}

Modulo a technical lemma, Lemma 5.6, we are ready to prove Theorem 1.1. Lemma 5.6 is hard to motivate on its own, so let us postpone it until we need it. 
Proof of Theorem 1.1 As mentioned earlier, we are proving this for $i=1$. Due to Theorem 4.2, we are free to choose our Heegaard diagram. So let $\mathcal{H}$ be an index- $n$ grid diagram representing the pointed link $(L, p)$; the Heegaard surface $\Sigma$ is a torus, so let us call it $T$; let $J_{s}$ be a constant path of almost complex structure on $\operatorname{Sym}^{n}(T)$ induced from some complex structure $\mathfrak{j}$ on $T$; as usual, let $w_{1,1}, z_{1,1}, \ldots, w_{1, k_{1}}, z_{1, k_{1}}$ be the markings, in order, on the oriented link component $L_{1}$, with $k_{1}>1$ and $w_{1,1}$ being the special $w_{1}$-marking. Later on, during the proof of Lemma 5.6, we will impose further restrictions on $\mathcal{H}$; but, for now, let us not be concerned with such restrictions.

As in Section 5.6, for $1 \leq j \leq k_{1}$ let $\mathcal{G}_{j}$ be the grid diagram where $w_{1, j}$ is the special $w_{1}$-marking but which is otherwise identical to $\mathcal{H}$; let $\left(L, p^{(j)}\right)$ be the pointed link that $\mathcal{G}_{j}$ represents (in particular, $\mathcal{G}_{1}=\mathcal{H}$ and $p^{(1)}=p$ ). For each $j$, starting at $\left(\mathcal{G}_{j}, J_{s}\right)$, we will do a stabilization, a sequence of horizontal commutations, a sequence of vertical commutations, a renumbering, a destabilization and finally a change of complex structure to obtain a diagram $\left(\mathcal{G}_{j+1}^{\prime}, J_{s}^{\prime}\right)$; we will then apply a self-homeomorphism $\tau_{j, j+1}$ of $T$ that sends $\mathcal{G}_{j+1}^{\prime}$ to $\mathcal{G}_{j+1}$ and $J_{s}^{\prime}$ to $J_{s}$ (the relevant subscripts are numbered modulo $k_{1}$ ). This process is best described by Figure 5.4 (the shaded region might contain additional $w$ - and $z$-markings).

Let us now discuss a minor subtlety that we had held back for this long. If we stabilize or destabilize a grid diagram (or a Heegaard diagram in general), the new diagram does not represent the same pointed link; it represents an isotopic link. However, we want the two diagrams to represent "nearby" links. Towards this end, let us broaden the class of links that a Heegaard diagram represents.

Given a grid diagram $\mathcal{G}=(T, \alpha, \beta, z, w)$, let $U_{\alpha}$ and $U_{\beta}$ be the handlebodies specified by the data $(T, \alpha)$ and $(T, \beta)$, respectively. To obtain $a$ link that $\mathcal{G}$ represents, in each component of $\Sigma \backslash \alpha$ join the $z$-marking to the $w$-marking by an embedded path and push the interior of the path into the interior of $U_{\alpha}$, and in each component of $\Sigma \backslash \beta$ in join the $w$-marking to the $z$-marking by an embedded path and push the interior of the path into the interior of $U_{\beta}$. If these "pushes" are small, then each of the links that $\mathcal{G}$ represents is supported in a small neighborhood of $T$. From now on, let us assume that the links represented by grid diagrams are supported in a small neighborhood of the Heegaard torus.

Therefore, when we stabilize a grid diagram $\mathcal{G}$ to get a grid diagram $\mathcal{G}^{\prime}$, even though the process changes the underlying links, the change is not drastic; the process merely introduces a "kink"; see Figure 5.5. Therefore, modulo introducing a small kink and then removing another, the self-homeomorphism $\tau_{j, j+1}$ of $T$ induces a selfhomeomorphism $\tilde{\tau}_{j, j+1}$ of $\left(S^{3}, L\right)$; it is constant outside a small neighborhood of the oriented arc that joins $p_{1}^{(j)}=w_{1, j}$ to $p_{1}^{(j+1)}=w_{1, j+1}$ on $L_{1}$ and it sends $p_{1}^{(j)}$ 


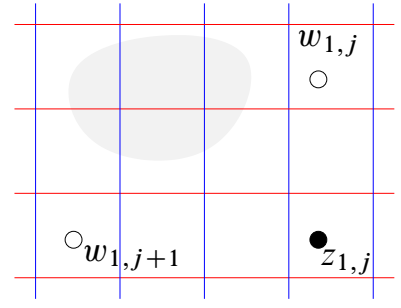

We start with the diagram $\left(\mathcal{G}_{j}, J_{s}\right)$.

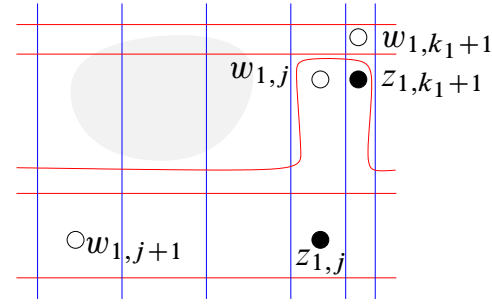

We do a sequence of horizontal commutations.

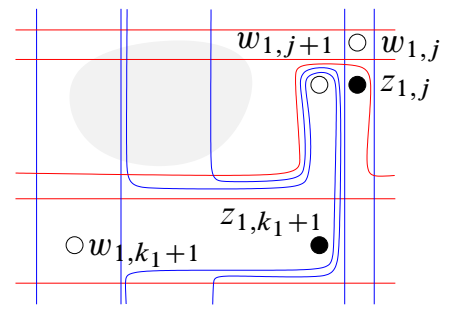

We renumber the $w$ - and $z$-markings.

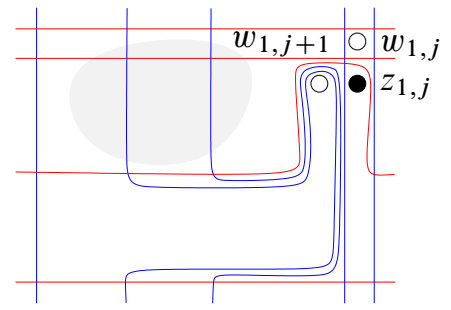

We change the complex structure on $T$ from $\mathfrak{j}$ to $\mathfrak{j}^{\prime}=\tau_{j, j+1}^{-1}(\mathfrak{j})$ to get the diagram $\left(\mathcal{G}_{j+1}^{\prime}, J_{s}^{\prime}\right)$.

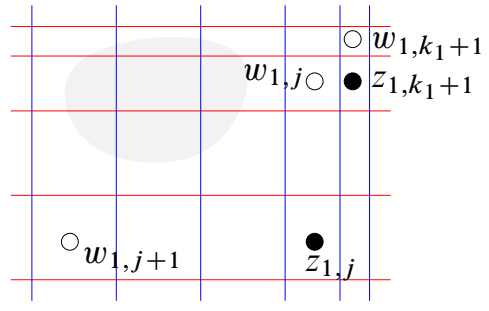

We stabilize by adding $w_{1, k_{1}+1}$ and $z_{1, k_{1}+1}$.

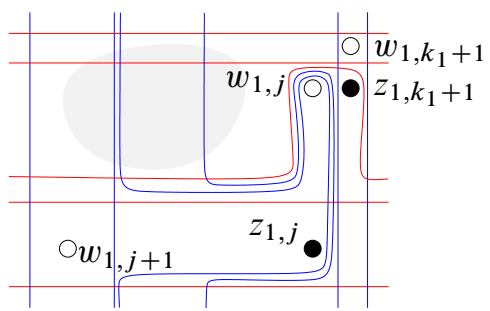

We do a sequence of vertical commutations.

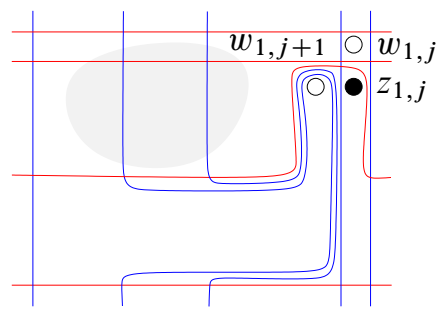

We destabilize by deleting $w_{1, k_{1}+1}$ and $z_{1, k_{1}+1}$. There is an obvious homeomorphism $\tau_{j, j+1}$ which converts this diagram to $\mathcal{G}_{j+1}$.

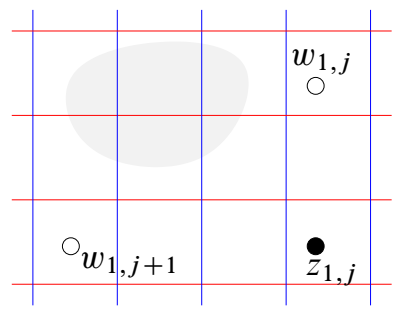

We apply the homeomorphism $\tau_{j, j+1}$ to get the final diagram $\left(\mathcal{G}_{j+1}, J_{s}\right)$.

Figure 5.4: The sequence of moves converting $\left(\mathcal{G}_{j}, J_{s}\right)$ to $\left(\mathcal{G}_{j+1}, J_{s}\right)$ 

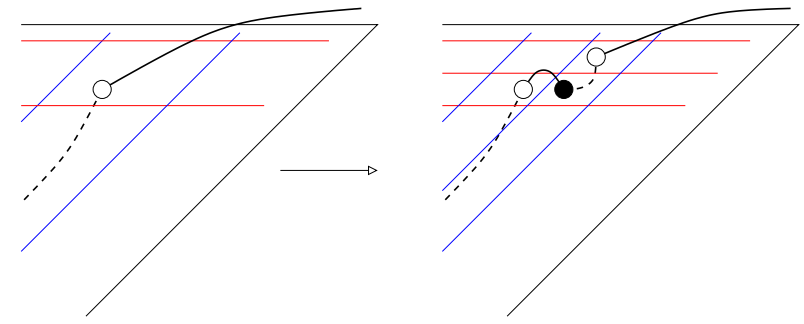

Figure 5.5: Stabilization introduces a kink in the link.

to $p_{1}^{(j+1)}$. Thus, the composition $\tilde{\tau}_{k_{1}, 1} \cdots \tilde{\tau}_{2,3} \tilde{\tau}_{1,2}$ induces the mapping class group element $\sigma_{1} \in \operatorname{MCG}\left(S^{3}, L, p\right)$.

The sequence of moves that converts $\left(\mathcal{G}_{j}, J_{s}\right)$ to $\left(\mathcal{G}_{j+1}^{\prime}, J_{s}^{\prime}\right)$ induces a map from $C F_{\left(\mathcal{G}_{j}, J_{s}\right)}$ to $C F_{\left(\mathcal{G}_{j+1}^{\prime}, J_{s}^{\prime}\right)}$ (the map is the composition of a stabilization map, some horizontal commutation maps, some vertical commutation maps, a renumbering map, a destabilization map and a change of complex structure map). The automorphism $\tau_{j, j+1}$ induces an identification between $C F_{\left(\mathcal{G}_{j+1}^{\prime}, J_{s}^{\prime}\right)}$ and $C F_{\left(\mathcal{G}_{j+1}, J_{s}\right)}$. The composition is an $\mathbb{F}_{2}\left[U_{1}, \ldots, U_{l}\right]$-module chain map from $C F_{\mathcal{G}_{j}}$ to $C F_{\mathcal{G}_{j+1}}$. Lemma 5.6 will prove that, for certain types of grid diagrams called superstabilized grid diagrams, this map is in fact the map $f_{j, j+1}=c_{j, j+1} \Phi_{1, j} \sum_{J \in J(j, j+1)} \Psi_{1, j}=c_{j, j+1} \Phi_{1, j} \Psi_{1, j}$.

A superstabilized grid diagram is a grid diagram where every $w$ - or $z$-marking lies in a $2 \times 2$ square $S$ which looks like one of the following: $S$ contains a $z$-marking at the southeast square and two $w$-markings, one each at the northeast square and the southwest square; or $S$ contains a $w$-marking at the northwest square and two $z-$ markings, one each at the northeast square and the southwest square. It is clear that any grid diagram can be converted to a superstabilized one by stabilizing sufficiently many times. Therefore, we may assume that our original grid diagram $\mathcal{H}$ was superstabilized to start with.

Lemma 5.6 Assuming that $\mathcal{G}_{j}$ is a superstabilized grid diagram, the sequence of moves from Figure 5.4, which converts $\left(\mathcal{G}_{j}, J_{s}\right)$ to $\left(\mathcal{G}_{j+1}, J_{s}\right)$, induces the map $f_{j, j+1}=$ $c_{j, j+1} \Phi_{1, j} \Psi_{1, j}$ from $C F_{\mathcal{G}_{j}}$ to $C F_{\mathcal{G}_{j}^{\prime}}$.

Proof Let $\mathcal{G}^{m}$ be the grid diagram shown in the $m^{\text {th }}$ picture of Figure 5.4 (hence, $\mathcal{G}^{0}=\mathcal{G}_{j}$ and $\left.\mathcal{G}^{8}=\mathcal{G}_{j+1}\right)$. The map induced by the sequence of moves is the composition of the following maps: a stabilization map $s$ from $C F_{\mathcal{G}^{1}}$ to $C F_{\mathcal{G}^{2}}$; the composition $h$ of some horizontal commutation maps, which maps from $C F_{\mathcal{G}^{2}}$ to $C F_{\mathcal{G}^{3}}$; the composition $v$ of some vertical commutation maps, which maps from $C F_{\mathcal{G}^{3}}$ to $C F_{\mathcal{G}^{4}}$; a renumbering map $r$ from $C F_{\mathcal{G}^{4}}$ to $C F_{\mathcal{G}^{5}}$; a destabilization map $d$ from $C F_{\mathcal{G}^{5}}$ 

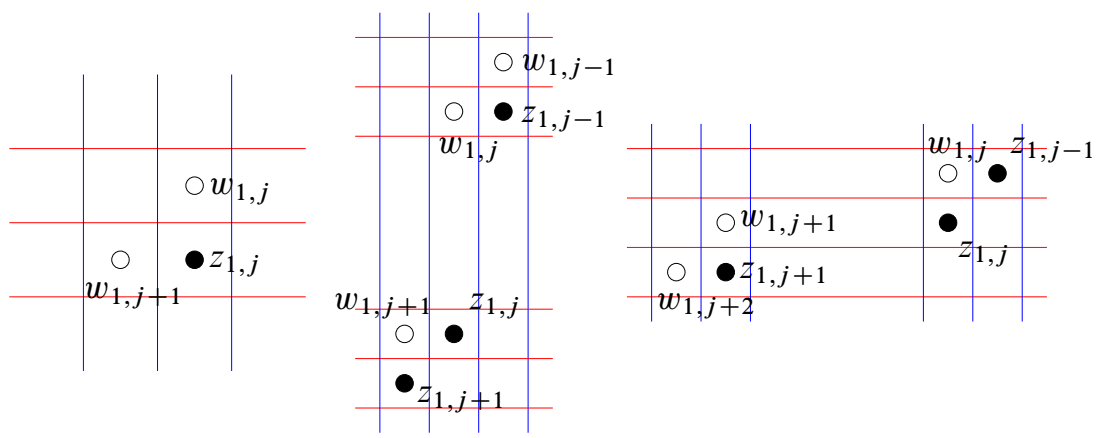

Figure 5.6: The three possible configurations of $\mathcal{G}_{j}$

to $C F_{\mathcal{G}^{6}}$; a change of complex structure map from $C F_{\mathcal{G}^{6}}$ to $C F_{\mathcal{G}^{7}}=C F_{\mathcal{G}^{6}}$, which is the identity map; and finally an identification map induced by $\tau_{j, j+1}$ from $C F_{\mathcal{G}^{7}}$ to $C F_{\mathcal{G}^{8}}$. Therefore, if we identify $C F_{\mathcal{G}^{6}}$ with $C F_{\mathcal{G}^{1}}$ by an identification map $\iota$, the map in question is simply the composition $\iota d r v h s$.

Since $\mathcal{G}_{j}$ is a superstabilized grid diagram, it actually looks like one of the three configurations of Figure 5.6. The first case is the easiest, so we will do it for warm-up. The second and the third case are similar, so we will only do the second case.

In the first case, $\mathcal{G}^{2}=\mathcal{G}^{3}=\mathcal{G}^{4}$ and $h=v=\mathrm{Id}$. The sequence of moves is shown in Figure 5.7. We want to show that the composition $\iota d r s$ equals the map $c_{j, j+1} \Phi_{1, j} \Psi_{1, j}$. It is easy to see that the part $c_{j, j+1}$ comes from renumbering map $r$ and the destabilization map $d$. We only have to check that the domains that contribute to the map $d r s$ correspond to the domains that contribute to the map $\Phi_{1, j} \Psi_{1, j}$.

Recall that the stabilization map $s$ comes from certain northeast snail domains and they are not allowed to pass through $z_{1, j}$; similarly, the destabilization map $d$ comes from certain southeast snail domains and they too are not allowed to pass through $z_{1, j}$. Therefore, only domains of certain "shapes" can appear in the map $d r s$ and these

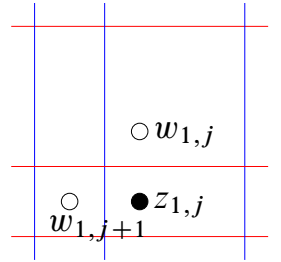

$\mathcal{G}^{1}$

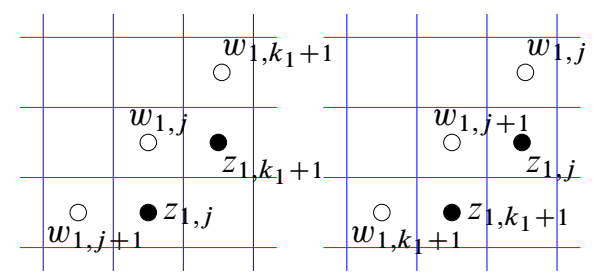

$\mathcal{G}^{5}$

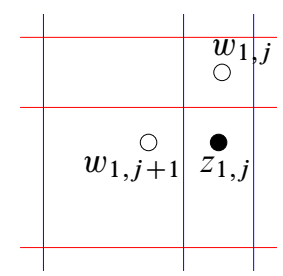

$\stackrel{d}{\triangleright}$

$\mathcal{G}^{6}$

Figure 5.7: The sequence of moves in the first case 

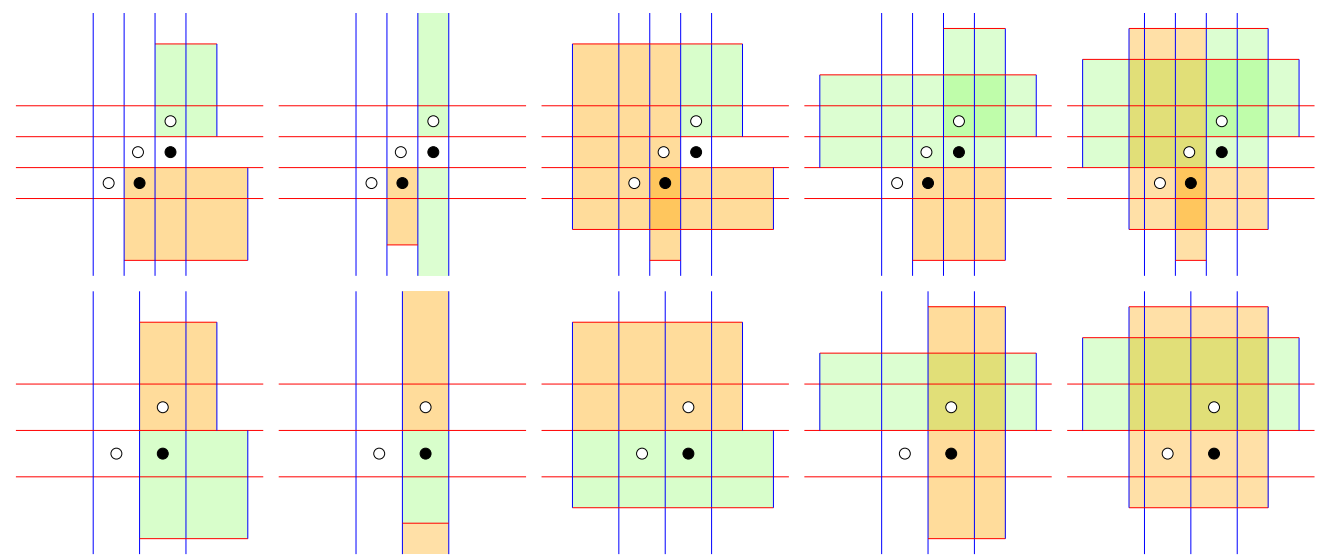

Figure 5.8: The correspondence of domains in the first case. The domains appearing in the map $d r s$ are shown in the top row and the corresponding domains appearing in $\Phi_{1, j} \Psi_{1, j}$ are shown in the bottom row. The domains corresponding to $s$ and $\Psi_{1, j}$ are shown in green, while the domains corresponding to $d$ and $\Phi_{1, j}$ are shown in orange.

shapes correspond to the domains that appear in the map $\Phi_{1, j} \Psi_{1, j}$. This is best illustrated by Figure 5.8.

In the second case, $\mathcal{G}^{3}=\mathcal{G}^{4}$ and $v=\mathrm{Id}$. The sequence of moves is shown in Figure 5.9. In this case, the maps $\iota d r h s$ and $c_{j, j+1} \Phi_{1, j} \Psi_{1, j}$ will not agree on the nose; we will have to modify the second map by a homotopy. Given states $x, y \in \mathcal{S}_{\mathcal{G}_{j}}$, let $\mathcal{R}_{H}(x, y)$ be the subset of $\mathcal{R}_{\mathcal{G}_{j}}(x, y)$ consisting of all the rectangles that contain both $w_{1, j}$ and $z_{1, j}$ with $z_{1, j}$ lying to the north of $w_{1, j}$. Define the $U_{l, j}$-equivariant map

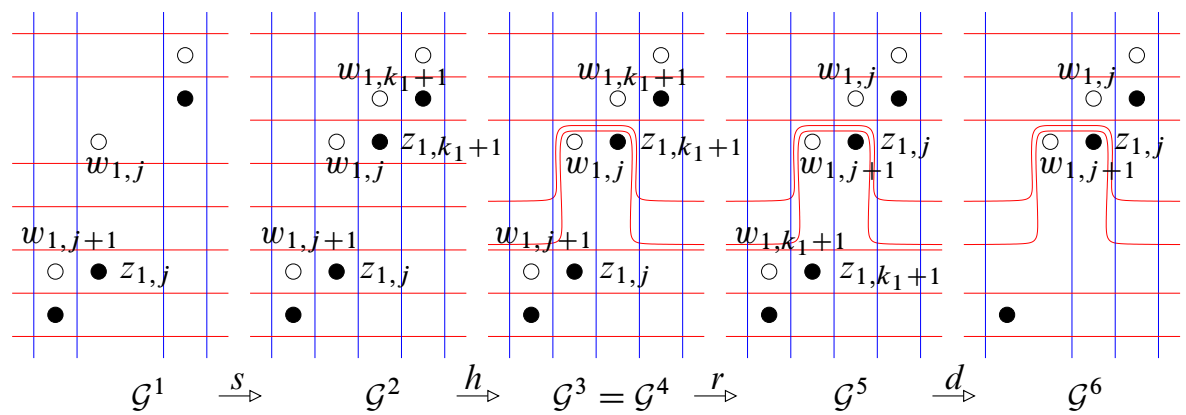

Figure 5.9: The sequence of moves in the second case. There could be arbitrarily many $\alpha$-circles between $w_{1, j}$ and $z_{1, j}$; we have drawn three. 
$H: C F_{\mathcal{G}_{j}} \rightarrow C F_{\mathcal{G}_{j}}$ as follows:

$$
H(x)=\sum_{y \in \mathcal{S}_{\mathcal{G}_{j}}} y U_{1, j}^{-1} \sum_{\substack{R \in \mathcal{R}_{H}(x, y) \\ n_{z}(R)=1}} \prod_{l, j} U_{l, j}^{n_{w_{l, j}}(R)} .
$$

The map $c_{j, j+1} H$ is a $U_{i}$-equivariant map from $C F_{\mathcal{G}_{j}}$ to $C F_{\mathcal{G}_{j+1}}$. Furthermore, $\left[c_{j, j+1} H: \partial\right]=\left(U_{1, j}+U_{1, j+1}\right) c_{j, j+1} \Phi_{1, j} H+c_{j, j+1}[H: \partial]$. However, it is easy to see that $\Phi_{1, j} H=0$ for the case at hand. We will show that the maps $\iota d r h s$ and $c_{j, j+1} \Phi_{1, j} \Psi_{1, j}+\left[c_{j, j+1} H: \partial\right]=c_{j, j}\left(\Phi_{1, j} \Psi_{1, j}+[H: \partial]\right)$ are equal. The part $c_{j, j+1}$ once again comes from $r$ and $d$. Therefore, we only have to show that the domains that contribute to the map $d r h s$ correspond to the domains that contribute to the map $\Phi_{1, j} \Psi_{1, j}+[H: \partial]$.

Recall that in a horizontal commutation we change a single $\alpha$-circle and the commutation map is given by counting certain pentagons, one of whose vertices is $\varrho$, which is one of the two intersection points between the old $\alpha$-circle and the new $\alpha$-circle. Therefore, such pentagons could be two types: the north pentagons, which lie to the north of $\varrho$, and the south pentagons, which lie to the south of $\varrho$. The map $h$ is the composition of several such commutation maps. Furthermore, it is clear that in the composition, a south pentagon can not be followed by a north pentagon. Therefore, any domain corresponding to the map $h$ is a sum of two domains: one coming from the north pentagons, followed by one coming from the south pentagons.

The north pentagons happen immediately after the stabilization map $s$; hence, there are only a few possible configurations for a domain corresponding to the north pentagons. Similarly, the south pentagons are immediately followed by the destabilization map $d$; therefore, there are only a few possible configurations for a domain corresponding to the south pentagons as well. This allows us to do a case analysis, whereby we can show that the domains contributing to the map $d r h s$ correspond to the domains contributing to the map $\Phi_{1, j} \Psi_{1, j}+[H: \partial]$; see Figure 5.10.

To complete the proof of Theorem 1.1, recall that the composition $\tilde{\tau}_{k_{1}, 1} \cdots \tilde{\tau}_{2,3} \tilde{\tau}_{1,2}$ induces the mapping class group element $\sigma_{1} \in \operatorname{MCG}\left(S^{3}, L, p\right)$. Therefore, the composition $f_{k_{1}, 1} \cdots f_{2,3} f_{1,2}$ must equal $\rho\left(\sigma_{1}\right)$. However, by Theorem 5.5,

$$
\begin{aligned}
f_{k_{1}, 1} f_{k_{1}-1, k_{1}} \cdots f_{2,3} f_{1,2}=f_{k_{1}, 1} f_{k_{1}-1, k_{1}} \cdots f_{1,3}=\cdots & =f_{k_{1}, 1} f_{1, k_{1}} \\
& =\operatorname{Id}+\Phi_{1} \Psi_{1} .
\end{aligned}
$$




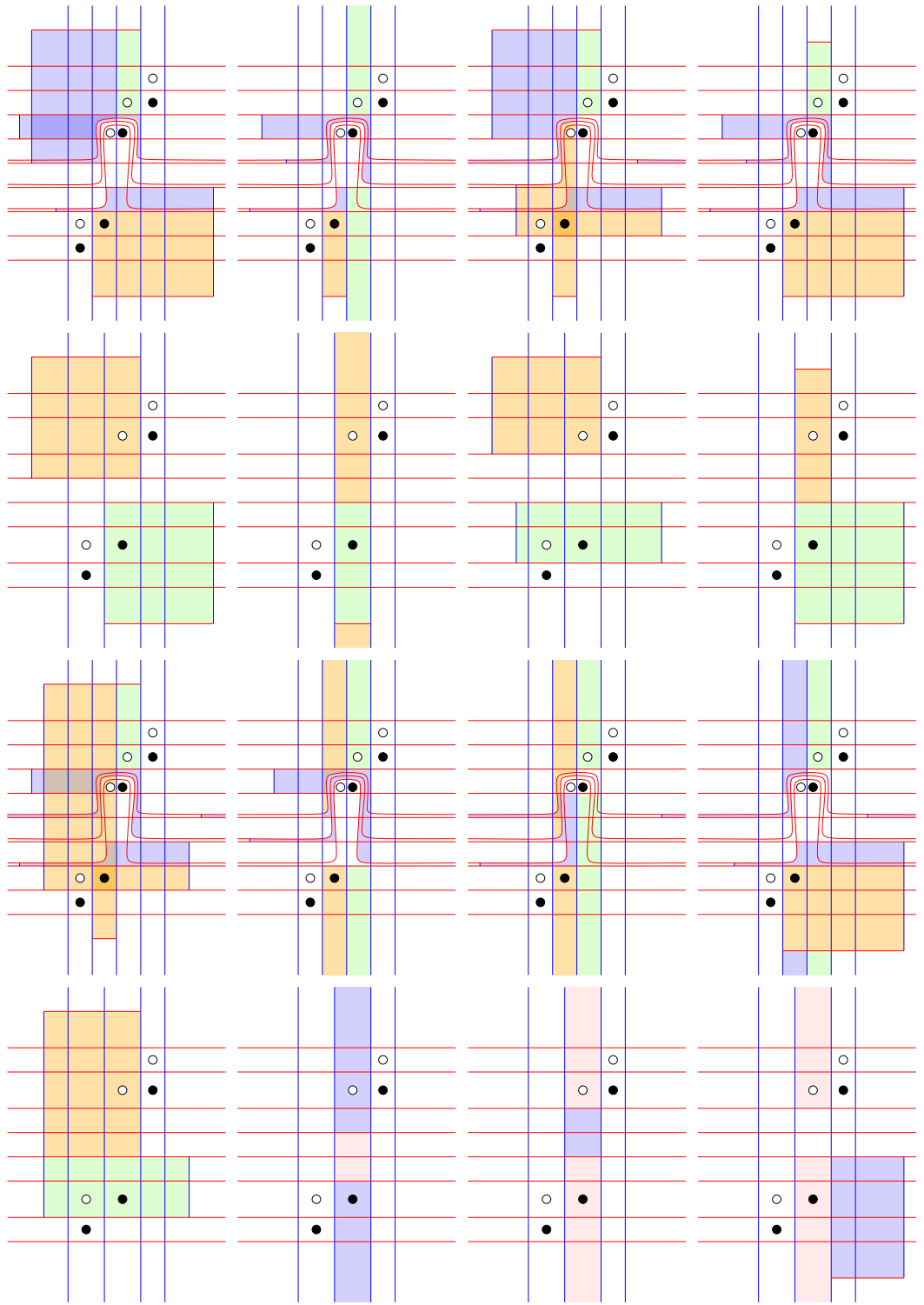

Figure 5.10: The correspondence of domains in the second case (here we have drawn four $\alpha$-circles between $w_{1, j}$ and $z_{1, j}$ ). The domains appearing in the map $d r h s$ are shown in the first and the third row and the corresponding domains appearing in $\Phi_{1, j} \Psi_{1, j}+[H: \partial]$ are shown in the second and the fourth row. The domains corresponding to $s$ and $\Psi_{1, j}$ are shown in green, the domains corresponding to $d$ and $\Phi_{1, j}$ are shown in orange, the domains corresponding to $h$ and the first map in $[H: \partial]$ are shown in violet, and the domains corresponding to the second map in $[H: \partial]$ are shown in pink. 


\section{Computations}

Let us now present some computations. We will only work with knots in $S^{3}$; since there is only one component to work with, we will write $\Phi$ and $\Psi$ instead of $\Phi_{1}$ and $\Psi_{1}$. For a pointed knot $(K, p)$, define the polynomials $h_{K}(q, t), r_{K}(q, t) \in \mathbb{Z}\left[q, q^{-1}, t, t^{-1}\right]$ as follows: the coefficient of $q^{a} t^{b}$ in $h_{K}(q, t)$ is the dimension (as an $\mathbb{F}_{2}$-module) of $\widehat{H F} L\left(S^{3}, K, p\right)$ in $(M, A)$-bigrading $(a, b)$; the coefficient of $q^{a} t^{b}$ in $r_{K}(q, t)$ is the rank of the map $\Phi \Psi$ on $\widehat{H F} L\left(S^{3}, K, p\right)$ in $(M, A)$-bigrading $(a, b)$. Since $(\Phi \Psi)^{2}=0$, the only information that we can get from it is its rank.

If a knot $K$ satisfies $h_{K}(q, t)=t^{s} \tilde{h}(q t)$ for some $s \in \mathbb{Z}$ and $\tilde{h} \in \mathbb{Z}\left[v, v^{-1}\right]$, it is called a thin knot; if, in addition, $s=-\frac{1}{2} \sigma(K)$, where $\sigma(K)$ is the signature of $K$, then $K$ is called $\sigma$-thin. The "Euler characteristic" of knot Floer homology is its symmetrized Alexander-Conway polynomial [11], ie $h_{K}(-1, t)=\Delta_{K}(t)$. Therefore, for $\sigma$-thin knots, the knot Floer homology can be reconstructed from the Alexander-Conway polynomial and the knot signature as $h_{K}(q, t)=(-q)^{\sigma(K) / 2} \Delta_{K}(-q t)$. All quasialternating knots are $\sigma$-thin $[10 ; 5]$ and, out of the 85 prime knots up to nine crossings, 83 are quasi-alternating. The following theorem shows that, for thin knots, $r_{K}(q, t)$ can be constructed from $h_{K}(q, t)$ and hence, for $\sigma$-thin knots, $r_{K}(q, t)$ can be constructed from the signature and the Alexander-Conway polynomial.

Theorem 6.1 If $K$ is a thin knot with $h_{K}(q, t)=t^{s} \tilde{h}(q t)$, then $r_{K}(q, t)=t^{s} \tilde{r}(q t)$, where $\tilde{r} \in \mathbb{Z}\left[v, v^{-1}\right]$ is the unique polynomial such that, for some $m \in \mathbb{Z}, \tilde{h}(v)=$ $\left(1-v^{2 m+1}\right) /(1-v)+\left(v^{-1}+2+v\right) \tilde{r}(v)$.

Proof The uniqueness is the easy part. If there are two different polynomials $\tilde{r}$ and $\tilde{r}^{\prime}$ that satisfy the above equation, then we get

$$
(1+v)^{2}(1-v)\left(\tilde{r}-\tilde{r}^{\prime}\right)=v^{2 m+2}-v^{2 m^{\prime}+2} .
$$

Differentiating once and then putting $v=-1$ shows that $m=m^{\prime}$ and hence $\tilde{r}=\tilde{r}^{\prime}$.

The knot Floer homology $\widehat{H F} L\left(S^{3}, K, p\right)$ is a graded object with the grading being the Maslov grading ${ }^{4}$ and, due to Lemma 4.4, it carries two commuting differentials $\Phi$ and $\Psi$ of gradings 1 and -1 , respectively. There are two knot-invariant spectral sequences: one starts at $\left(\widehat{H F} L\left(S^{3}, K, p\right), \Phi\right)$ and converges to $\mathbb{F}_{2}$, where the differentials on the $i^{\text {th }}$ page shift the $(M, A)$-bigrading by $(2 i-1, i)$; and the second one starts at $\left(\widehat{H F} L\left(S^{3}, K, p\right), \Psi\right)$ and converges to $\mathbb{F}_{2}$ lying in Maslov grading 0 , where the differentials on the $i^{\text {th }}$ page shift the $(M, A)$-bigrading by $(-1,-i)$. Therefore, for thin knots, both the spectral sequences must collapse immediately, ie

\footnotetext{
${ }^{4}$ Since we are working with a thin knot, the Alexander grading differs from this grading by a constant.
} 
$H_{*}\left(\widehat{H F} L\left(S^{3}, K, p\right), \Phi\right)=\mathbb{F}_{2}$ and $H_{*}\left(\widehat{H F} L\left(S^{3}, K, p\right), \Psi\right)=\mathbb{F}_{2}$. Therefore, from a slight generalization of [16, Lemma 7], ${ }^{5}$ we see that $\left(\hat{H F} L\left(S^{3}, K, p\right), \Phi, \Psi\right)$ must be isomorphic to a direct sum of square pieces of the form $\left(\mathbb{F}_{2}\left(a_{1}, a_{2}, a_{2}^{\prime}, a_{3}\right), \Phi, \Psi\right)$, where $\Phi\left(a_{1}\right)=\Psi\left(a_{3}\right)=a_{2}^{\prime}, \Phi\left(a_{2}\right)=a_{3}$ and $\Psi\left(a_{2}\right)=a_{1}$, and a single ladder piece of the form $\left(\mathbb{F}_{2}\left(b_{1}, \ldots, b_{2 m+1}\right), \Phi, \Psi\right)$, where either $\Phi\left(b_{2 i-1}\right)=\Psi\left(b_{2 i+1}\right)=b_{2 i}$ for all $1 \leq i \leq m$ or $\Phi\left(b_{2 i}\right)=b_{2 i+1}$ and $\Psi\left(b_{2 i}\right)=b_{2 i-1}$ for all $1 \leq i \leq m$. The only contributions to the map $\Phi \Psi$ come from the square pieces in the middle grading, namely $\Phi \Psi\left(a_{2}\right)=a_{2}^{\prime}$. This produces the required decomposition of $\tilde{h}(v)$ : the square pieces contribute the term $\left(v^{-1}+2+v\right) \tilde{r}(v)$; the ladder piece contributes the term $1+\cdots+v^{2 m}$ in the first case and the term $1+\cdots+v^{-2 m}$ in the second case.

The two prime knots up to nine crossings that are not thin (and hence not quasialternating) are $8_{19}$ and $9_{42}$.

Lemma 6.2 $r_{8_{19}}(q, t)=0$ and $r_{9_{42}}(q, t)=t^{-1}+q^{2} t$.

Proof From [1], we get

$$
\begin{aligned}
& h_{8_{19}}(q, t)=t^{-3}+q t^{-2}+q^{2}+q^{5} t^{2}+q^{6} t^{3}, \\
& h_{9_{42}}(q, t)=q^{-1} t^{-2}+2 t^{-1}+1+2 q+2 q^{2} t+q^{3} t^{2} .
\end{aligned}
$$

Since $(\Phi \Psi)^{2}=0$, the coefficient of $q^{a} t^{b}$ in $r_{K}(q, t)$ is less than or equal to half the coefficient of $q^{a} t^{b}$ in $h_{K}(q, t)$. This immediately shows that $r_{8_{19}}(q, t)=0$.

The knot Floer homology of $9_{42}$ is supported on two diagonals, $M-A=0$ and $M-A=1$. For $i \in\{0,1\}$, let $C_{i}$ be the direct summand that is supported in the diagonal $M-A=i$. As in the proof of Theorem 6.1, there are two knot-invariant spectral sequences starting at $\left(C_{1}, \Phi\right) \oplus\left(C_{0}, \Phi\right)$ and $\left(C_{1}, \Psi\right) \oplus\left(C_{0}, \Psi\right)$ and ending at $\mathbb{F}_{2}$. By [1], the first spectral sequence collapses at the very next step, ie $H_{*}\left(C_{1}, \Phi\right)=0$. Since the knot $9_{42}$ is reversible and the spectral sequences are knot invariants, the second spectral sequence must also collapse at the very next step, ie $H_{*}\left(C_{1}, \Psi\right)=0$. Another application of [16, Lemma 7] tells us that $\left(C_{1}, \Phi, \Psi\right)$ is a direct sum of two square pieces and hence $r_{9_{42}}(q, t)=t^{-1}+q^{2} t$.

We conclude with the following observation; this can independently be proved by more classical tools, such as studying combinatorial symmetries of the ideal cell decomposition of the knot complements, which in turn is easily computed by computer programs like SnapPea (M Thistlethwaite, personal communication).

\footnotetext{
${ }^{5}$ In [16], they do it for bigraded complexes; however, the proof goes through for singly graded complexes like the one under consideration.
} 
Theorem 6.3 There does not exist any orientation-preserving involution of $S^{3}$ that acts freely and in an orientation-preserving way on either the knot $8_{20}$ or the knot $9_{42}$.

Proof Let $(K, p)$ be a pointed oriented knot in $S^{3}$ and let $\tau$ be an orientationpreserving involution of $S^{3}$ that acts freely and in an orientation-preserving way on $K$. Let $\bar{\sigma}$ be half a Dehn twist around $K$ that interchanges the points $p$ and $\tau(p)$ such that $\bar{\sigma}^{2}=\sigma$, the full positive Dehn twist around $K$. Since $\tau$ acts freely on a neighborhood of $K$ and $\bar{\sigma}$ is the identity outside a neighborhood of $K$, the two diffeomorphisms $\tau \bar{\sigma}$ and $\bar{\sigma} \tau$ induce the same element of $\operatorname{MCG}\left(S^{3}, K, \tau(p)\right)$; hence, the two diffeomorphisms $(\tau \bar{\sigma})^{2}$ and $\tau^{2} \bar{\sigma}^{2}=\sigma$ induce the same element in $\operatorname{MCG}\left(S^{3}, K, p\right)$.

Let $\hat{\rho}: \operatorname{MCG}\left(S^{3}, K, p\right) \rightarrow \operatorname{Aut}_{N\left(\mathcal{C}_{1}\right)}\left(\widehat{H F} L\left(S^{3}, K, p\right)\right)$ be the action of the mapping class group on knot Floer homology. Theorem 1.1 tells us that $\hat{\rho}(\sigma)=\operatorname{Id}+\Phi \Psi$. However, since $(\tau \bar{\sigma})^{2}=\sigma$ in the mapping class group, $\hat{\rho}(\sigma)=\operatorname{Id}+\Phi \Psi=(\hat{\rho}(\tau \bar{\sigma}))^{2}$.

We know from [1], Theorem 6.1 and Lemma 6.2 that

$$
\begin{aligned}
& h_{8_{20}}(q, t)=q^{-2} t^{-2}+2 q^{-1} t^{-1}+3+2 q t+q^{2} t^{2}, \\
& r_{8_{20}}(q, t)=q^{-1} t^{-1}+q t, \\
& h_{9_{42}}(q, t)=q^{-1} t^{-2}+2 t^{-1}+1+2 q+2 q^{2} t+q^{3} t^{2}, \\
& r_{9_{42}}(q, t)=t^{-1}+q^{2} t .
\end{aligned}
$$

For the knot $8_{20}$ in $(M, A)$-bigrading $(1,1)$, and for the knot $9_{42}$ in $(M, A)$-bigrading $(2,1)$, the homology is two-dimensional and the rank of the map $\Phi \Psi$ is one. Therefore, in either case, we can do a change of basis to represent the map $\operatorname{Id}+\Phi \Psi$ by the matrix

$$
\left(\begin{array}{ll}
1 & 1 \\
0 & 1
\end{array}\right) \text {. }
$$

However, this matrix is not a square in $\mathrm{GL}_{2}\left(\mathbb{F}_{2}\right)$.

\section{References}

[1] J A Baldwin, W D Gillam, Computations of Heegaard-Floer knot homology, J. Knot Theory Ramifications 21 (2012) 1250075, 65 MR2925428

[2] A Juhász, Cobordisms of sutured manifolds, preprint (2010) arXiv:0910.4382v2

[3] A Juhász, D Thurton, Naturality and mapping class groups in Heegaard Floer homology, preprint (2015) arXiv:1210.4996

[4] R Lipshitz, A cylindrical reformulation of Heegaard Floer homology, Geom. Topol. 10 (2006) 955-1097 MR2240908 
[5] C Manolescu, P Ozsváth, On the Khovanov and knot Floer homologies of quasialternating links, from: "Proceedings of the $14^{\text {th }}$ Gökova Geometry-Topology Conference”, (S Akbulut, T Önder, R J Stern, editors), International Press, Cambridge, MA (2008) 60-81 MR2509750

[6] C Manolescu, P Ozsváth, Heegaard Floer homology and integer surgeries on links, preprint (2011) arXiv:1011.1317

[7] C Manolescu, P Ozsváth, S Sarkar, A combinatorial description of knot Floer homology, Ann. of Math. 169 (2009) 633-660 MR2480614

[8] C Manolescu, P Ozsváth, Z Szabó, D Thurston, On combinatorial link Floer homology, Geom. Topol. 11 (2007) 2339-2412 MR2372850

[9] P Ozsváth, A I Stipsicz, Contact surgeries and the transverse invariant in knot Floer homology, J. Inst. Math. Jussieu 9 (2010) 601-632 MR2650809

[10] P Ozsváth, Z Szabó, Heegaard Floer homology and alternating knots, Geom. Topol. 7 (2003) 225-254 MR1988285

[11] P Ozsváth, Z Szabó, Holomorphic disks and knot invariants, Adv. Math. 186 (2004) 58-116 MR2065507

[12] P Ozsváth, Z Szabó, Holomorphic disks and three-manifold invariants: Properties and applications, Ann. of Math. 159 (2004) 1159-1245 MR2113020

[13] P Ozsváth, Z Szabó, Holomorphic disks and topological invariants for closed threemanifolds, Ann. of Math. 159 (2004) 1027-1158 MR2113019

[14] P Ozsváth, Z Szabó, Holomorphic triangles and invariants for smooth four-manifolds, Adv. Math. 202 (2006) 326-400 MR2222356

[15] P Ozsváth, Z Szabó, Holomorphic disks, link invariants and the multi-variable Alexander polynomial, Algebr. Geom. Topol. 8 (2008) 615-692 MR2443092

[16] I Petkova, Cables of thin knots and bordered Heegaard Floer homology, Quantum Topol. 4 (2013) 377-409 MR3134023

[17] J A Rasmussen, Floer homology and knot complements, PhD thesis, Harvard University (2003) MR2704683 Available at http://search.proquest.com/docview/ 305332635

[18] S Sarkar, Maslov index formulas for Whitney n-gons, J. Symplectic Geom. 9 (2011) 251-270 MR2811652

[19] S Sarkar, J Wang, An algorithm for computing some Heegaard Floer homologies, Ann. of Math. 171 (2010) 1213-1236 MR2630063

Department of Mathematics, Princeton University

Fine Hall, Washington Rd, Princeton, NJ 08544, USA

sucharit@math.princeton.edu

http://web.math.princeton.edu/ sucharit/

Received: 18 September $2011 \quad$ Revised: 25 June 2014 
